\title{
Theoretical and computational issues in modelling material failure in strong discontinuity scenarios
}

\author{
J. Oliver ${ }^{\mathrm{a}, *}$, A.E. Huespe ${ }^{\mathrm{b}}$ \\ ${ }^{a}$ E.T.S. Enginyers de Camins, Canals i Ports, Technical University of Catalonia (UPC), Campus Nord UPC (mòdul C-1), \\ Jordi Girona 3, 08034 Barcelona, Spain \\ ${ }^{\mathrm{b}}$ CIMEC/CONICET-UNL, Santa Fe, Argentina
}

Received 5 March 2003; received in revised form 15 July 2003; accepted 26 August 2003

\begin{abstract}
The paper deals with several aspects related to numerical modelling of material failure in strong discontinuity settings: (a) the onset and development of local material failure in terms of continuum constitutive models equipped with strain softening. Closed forms formulas for the solutions of the discontinuous material bifurcation problem are given for a class of those models; (b) finite elements with embedded discontinuities: nodal and elemental enrichments families are formulated in the continuum strong discontinuity approach (CSDA); (c) instability treatment: a discrete viscous perturbation method at the failure surfaces is presented as a way to substantially improve the robustness of the numerical simulations.
\end{abstract}

(C) 2004 Elsevier B.V. All rights reserved.

Keywords: Material failure; Continuum models; Strong discontinuities; Numerical modelling

\section{Motivation}

Material failure has been traditionally characterized by means of local concepts. In early stages, techniques and methodologies to detect and prevent material failure were grounded on the development of material failure indicators that provided safe limits to the range of stable structural behavior. Onset of inelasticity or loss of strong ellipticity have been classical failure indicators to characterize the beginning of more complex material behaviors that lead to the progressive softening of the structural response and, eventually, to the structural collapse. The most conservative methodologies were based on identifying the load level that activates, for the first time, those failure indicators at any of the material points of the structure as the critical load level or failure load, from which the design should move away through appropriate safety factors. However, it is nowadays very well known that, in ductile materials, loss of elasticity does not mean immediate local failure as material hardening follows. Most important, in many cases local material failure does not imply global structural failure since relevant zones of the structure must

\footnotetext{
${ }^{*}$ Corresponding author. Fax: +34-93-401-1048.

E-mail addresses: xavier.oliver@upc.es, oliver@cimne.upc.es (J. Oliver).
} 
fail locally before the structural failure (structural instability) can be observed. On the other hand the concept of critical load, as responsible for the structural instability, is relative to the loading pattern. Indeed, a limit point of the equilibrium path under prescribed load can became a stable point upon displacement control and make physically attainable postcritical states unreachable under load control. These facts have placed the present attention of material failure mechanics beyond the limits of the elastic range and the initiation of local failure. In fact, the aim of modern material failure mechanics is to identify as closely as possible the actual mechanisms of structural failure and to model the postcritical structural behavior.

It is also a well-accepted fact that global material failure under quasi-static loads for ductile and quasibrittle materials is characterized, from a macroscopical point of view, by the onset and propagation of failure lines (in 2D) or failure surfaces (in 3D). Local failure at a material point (or at a discrete set of points) propagates through the body along those failure surfaces, which are termed cracks, fractures, shear bands, or slip lines depending on the context. In many cases, the mechanism of formation of those failure locus can be explained as an initial local failure process followed by the concentration of the strains in a narrow band responsible for an increasing dissipation, which eventually collapses into a physically observable failure surface that propagates exhibiting a discontinuity in the displacement field. These displacement discontinuities will be from now on termed strong discontinuities and an approach to capture and to model them in a continuum (Continuum Mechanics) setting has been presented in detail elsewhere [19].

In this paper several theoretical and numerical aspects in modelling material failure in strong discontinuity settings are addressed. After a brief introduction of a strong discontinuity scenario in Section 2, the material bifurcation problem is tackled in Section 3 and an incursion is made into analytical, exact and
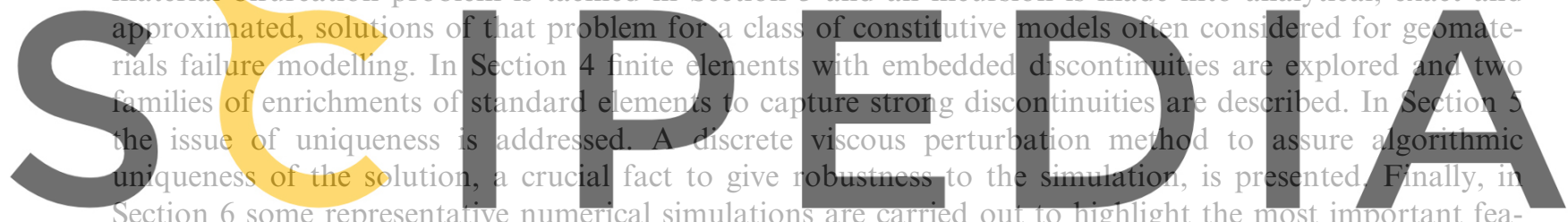

tures of the presented approach.

Register for free at https//www.scipedia.com to download the version without the watermark

\section{Strong discontinuity scenario}

In the last years the so-called strong discontinuity approach to material failure has been object of increasing attention. Here we consider an specific version faithful to the spirit of the pioneering work [33] and subsequently developed in [19-26]. It has been termed the continuum strong discontinuity approach (CSDA) since it features a continuum format even for purposes of modelling displacement discontinuities. The distinctive features of this approach, compared to alternative procedures that do not keep that continuum format $[1,2,12,34]$ have been pointed out elsewhere [24-26] and its main ingredients (that are explored in detail in [21]) can be summarized as follows:

1. The use of a continuum format for the kinematics, i.e.: the strains $\boldsymbol{\varepsilon}(\mathbf{x}, t)$ are computed as the symmetric gradient $\left(\nabla^{\mathrm{s}} \mathbf{u}\right)$ of the displacement field $\mathbf{u}(\mathbf{x}, t)$, even as it exhibits jumps across the failure path and, therefore, becomes unbounded (see Fig. 1). The displacement and strain fields read:

$$
\begin{aligned}
& \mathbf{u}(\mathbf{x}, t)=\overline{\mathbf{u}}(\mathbf{x}, t)+H_{S}[[\mathbf{u}]](\mathbf{x}, t), \\
& \boldsymbol{\varepsilon}(\mathbf{x}, \mathbf{t})=\nabla^{\mathrm{s}} \mathbf{u}=\underbrace{\overline{\boldsymbol{\varepsilon}}}_{\begin{array}{c}
\text { regular } \\
\text { (bounded) }
\end{array}}+\underbrace{\delta_{S}([[\mathbf{u}]] \otimes \mathbf{n})^{\mathrm{s}}}_{\begin{array}{c}
\text { singular } \\
\text { (unbounded) }
\end{array}},
\end{aligned}
$$




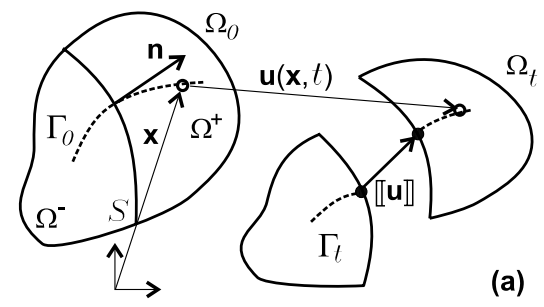

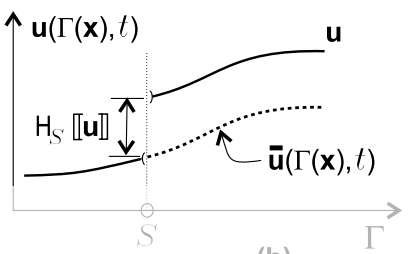

(b)

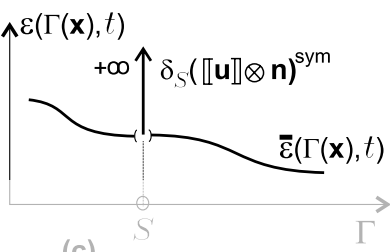

(c)

Fig. 1. Strong discontinuity kinematics: (a) initial and actual configurations of body $\Omega$; (b) displacement field; (c) strain field along the curve $\Gamma$.

where $H_{S}$ and $\delta_{S}$ stand, respectively, for the line/surface Heaviside's step function and Dirac's delta function placed at the discontinuity interface $S$ in the body $\Omega$, $\bar{u}$ is the regular (continuous) displacement

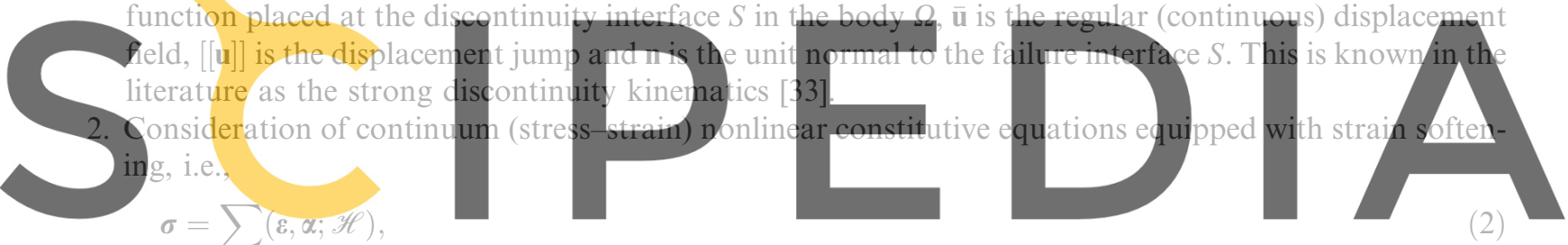

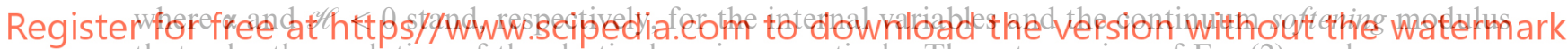

that rules the evolution of the elastic domain respectively. The rate version of Eq. (2) reads

$\dot{\sigma}=\mathbb{E}^{\operatorname{tang}}(\sigma, \alpha ; \mathscr{H}): \dot{\boldsymbol{\varepsilon}}$,

where $\mathbf{E}^{\text {tang }}$ is the tangent constitutive tensor.

3. The distributional character of the inverse of the continuum softening modulus $\mathscr{H}$ in terms of the discrete softening modulus $\overline{\mathscr{H}}$, i.e.,

$$
\frac{1}{\mathscr{H}}=\delta_{S} \frac{1}{\overline{\mathscr{H}}} \text {. }
$$

Regularized versions (more suitable for computational treatments) of Eqs. (1) and (4) are obtained via a $k$-regularized sequence of Dirac's delta functions:

$$
\delta_{S}^{k}(\mathbf{x})=\lim _{k \rightarrow 0} \mu_{S}(\mathbf{x}) \frac{1}{k},
$$

where $\mu_{S}$ is the collocation function on $S\left(\mu_{S}(\mathbf{x})=1\right.$ for $\mathbf{x} \in S$ and $\mu_{S}(\mathbf{x})=0$ otherwise) and $k$ is a regularization parameter as small as permitted by the machine precision. In this context Eq. (4) turns out to be the so-called softening modulus regularization condition [21]:

$$
\mathscr{H}(\mathbf{x})=k \overline{\mathscr{H}} \quad \forall \mathbf{x} \in S .
$$

The discrete softening modulus $\overline{\mathscr{H}}$ in Eq. (6) can be related to material properties as the fracture energy $G_{\mathrm{f}}$, Young's modulus $E$ and the uniaxial peak stress $\sigma_{\mathrm{u}}$, typically for linear softening: 


$$
\overline{\mathscr{H}}=-\frac{1}{2} \frac{\sigma_{\mathrm{u}}^{2}}{E G_{\mathrm{f}}}
$$

which qualifies $\overline{\mathscr{H}}$ as an actual material property from which the continuum softening parameter $\mathscr{H}$ at the failure interface can be obtained via Eq. (6).

In this context it can be shown [21] that:

(a) Eq. (4) (or its regularized counterpart (6)) makes compatible, for the constitutive equation (2), bounded values of the stresses $\boldsymbol{\sigma}$ with the unbounded strains $\boldsymbol{\varepsilon}_{S}$ emerging from Eq. (1) at the failure surface $S$.

(b) As the strong discontinuity kinematics (1) is activated, the original continuum constitutive equation (2) is projected into a discrete constitutive model (relating the traction at the failure interface $\mathscr{T}=\sigma_{S} \cdot \mathbf{n}$ with the displacement jump $\left.[[\mathbf{u}]]\right)$ that is automatically fulfilled at that failure interface [21], i.e.,

$$
\mathscr{T}=\mathscr{F}([[\mathbf{u}]], \overline{\mathscr{H}}) .
$$

Eq. (8) provides a clear link with the nonlinear decohesive Fracture Mechanics [11] while keeping the continuum format of the analysis.

4. Boundedness of the stress (and rate of stress) field and the traction-separation law (8) can only be achieved at the strong discontinuity regime if some restrictions on the stress field $\sigma_{S}$ at the discontinuity interface are fulfilled. These restrictions depend on the type of the parent constitutive model but they can
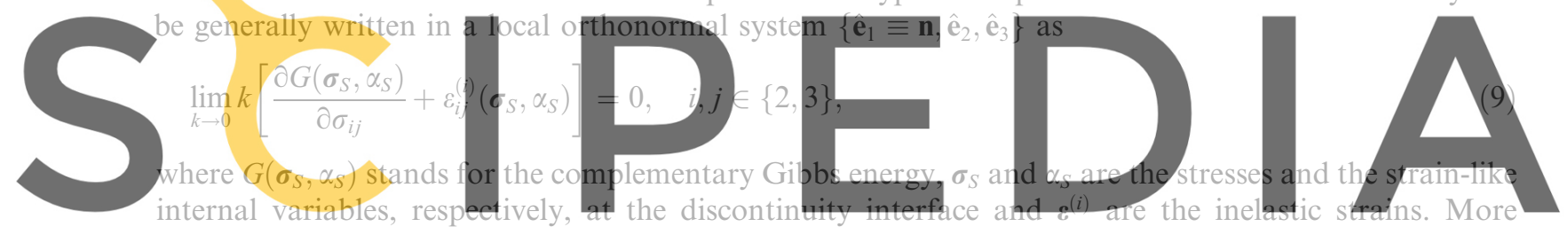

details on derivation of the strong discontinuity conditions (9) and their explicit form for particular

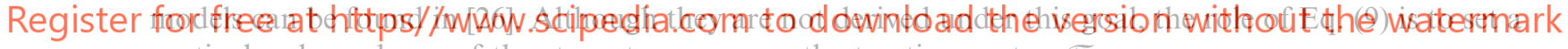

particular dependence of the stress tensor $\sigma_{S}$ on the traction vector $\mathscr{T}$ :

$$
\boldsymbol{\sigma}_{S}=\Sigma_{S}(\mathscr{T})
$$

this being ultimately responsible for the aforementioned projection of the continuum model (2) into the discrete one (8).

5. Since Eqs. (9) are not generally fulfilled, for a particular material point, at the onset of the material failure, a smooth transition to fulfillment of those conditions can be achieved by introducing a variable bandwidth model that characterizes a strong discontinuity as the ultimate collapse of a weak discontinuity $(h \equiv k \rightarrow 0)$ [24]. In physical terms, the dissipation phenomena taking place during that transition match the Fracture Process Zone concept in Fracture Mechanics [6,14].

\section{Onset and propagation of failure-closed form material bifurcation solutions}

Discontinuous bifurcation analyses $[28,30,35]$ provide a rigorous methodology to detect the onset of failure, in a given material point, as the loss of strong ellipticity of the tangent constitutive operator $\mathbf{E}^{\text {tang }}$, resulting in a local bifurcation of the stress-strain field. That bifurcation can be characterized in terms of the singularity of the so-called localization tensor:

$$
\mathbf{Q}^{\text {loc }}(\mathbf{x}, \mathbf{n}, \mathscr{H}, t)=\mathbf{n} \cdot \mathbf{E}^{\text {tang }}(\boldsymbol{\sigma}(\mathbf{x}, t), \mathscr{H}) \cdot \mathbf{n} .
$$


Therefore the bifurcation time $t_{\mathrm{B}}$, that triggers the discontinuous kinematics (1), is determined as the first time that the following condition is fulfilled for some $\mathbf{n}$ :

$$
\operatorname{det}\left[\mathbf{Q}^{\mathrm{loc}}\left(\mathbf{n}, \mathscr{H}, t_{\mathrm{B}}\right)\right]=0 .
$$

Eq. (12) implicitly defines, at the bifurcation time and for the given material point, the value of the softening modulus $\mathscr{H}=\mathrm{H}(\mathbf{n})$ as a function of the directions $\mathbf{n}$ fulfilling that equation. Then the critical value of the softening modulus $\mathscr{H}^{\text {crit }}$ and the (normal to the) propagation direction $\mathbf{n}^{\text {crit }}$ are determined by imposing $\mathscr{H}$ to be a maximum:

$$
\begin{aligned}
& \left.\mathbf{n}^{\text {crit }}=\underset{|\mathbf{n}|=1}{\arg [\max } \mathrm{H}(\mathbf{n})\right], \\
& \mathscr{H}^{\text {crit }}=\mathrm{H}\left(\mathbf{n}^{\text {crit }}\right) .
\end{aligned}
$$

In the context of modelling of material failure, Eqs. (13) can be solved numerically by a local maximization process [27]. However, for large problems, specially for 3D cases, this involves a large computational cost. For 2D (plane stress-plane strain) cases those equations have been very often solved by analytical procedures for particular families of continuum constitutive equations (plasticity, damage etc.). In [35] a geometrical interpretation of Eqs. (13), for a particular two-invariant parabolic plasticity model, invoked an appealing geometrical interpretation to solve those equations in Mohr's circle space. In the following sections a generalization of that methodology, through a similar but slightly different geometrical inter-

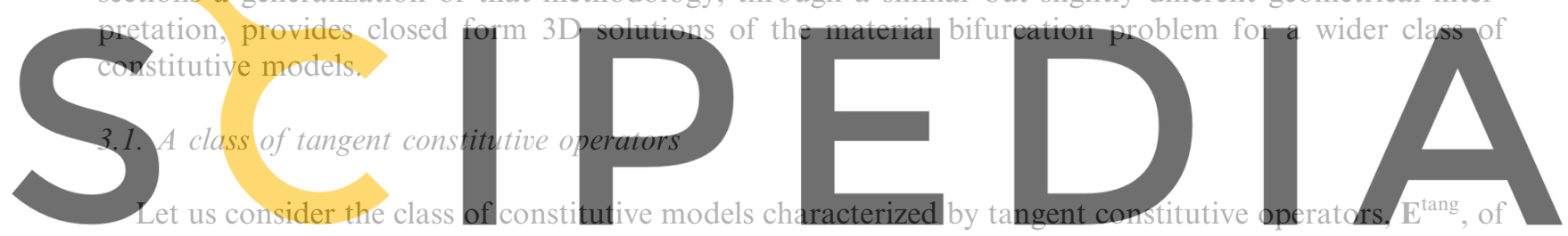

the form:

Register for free at https/fwww.scipedia.com to download the version without the watermark

$$
\boldsymbol{\sigma}=\mathbf{E}^{\text {tang }}: \dot{\varepsilon},
$$

where $\mathbf{E}^{*}$ is a fourth-order tensor, $\mathbf{P}$ and $\mathbf{R}$ are second-order symmetric tensors and $\xi(\mathscr{H})$ a scalar function of the softening modulus $\mathscr{H}$. The following conditions are required to those entities:

(1) The structure of $\mathbf{E}^{*}$ is such that its $\mathbf{n}$-projection $\mathbf{Q}^{*}(\mathbf{n}) \stackrel{\text { def }}{=} \mathbf{n} \cdot \mathbf{E}^{*} \cdot \mathbf{n}$ is the following:

$$
\begin{aligned}
& {\left[\mathbf{Q}^{*}(\mathbf{n})\right]^{-1}=-a \mathbf{n} \otimes \mathbf{n}+b \mathbf{1},} \\
& a>0 ; \quad b>0 ; \quad b-a>0 .
\end{aligned}
$$

(2) $\mathbf{P}$ and $\mathbf{R}$ are collinear and $\mathbf{P} \cdot \mathbf{R}$ is positive semi-definite. Therefore $\mathbf{P}, \mathbf{R}$ and $\mathbf{P} \cdot \mathbf{R}$ diagonalize in the same basis and eigenvalues of $\mathbf{P}\left(p_{1}, p_{2}, p_{3}\right)$ and eigenvalues of $\mathbf{R}\left(r_{1}, r_{2}, r_{3}\right)$ fulfill:

$$
\begin{aligned}
& p_{1} r_{1} \geqslant 0 \Rightarrow \operatorname{sign}\left(p_{1}\right)=\operatorname{sign}\left(r_{1}\right), \\
& p_{2} r_{2} \geqslant 0 \Rightarrow \operatorname{sign}\left(p_{2}\right)=\operatorname{sign}\left(r_{2}\right), \\
& p_{3} r_{3} \geqslant 0 \Rightarrow \operatorname{sign}\left(p_{3}\right)=\operatorname{sign}\left(r_{3}\right) .
\end{aligned}
$$

(3) $\xi(\mathscr{H}) \geqslant 0$ is the so-called generalized plastic modulus, a monotonous increasing function of the softening modulus $\mathscr{H}$. 
Remark 1. For the particular case of $\mathbf{E}^{*}=\mathbf{E}=\lambda \mathbf{1} \otimes \mathbf{1}+2 \boldsymbol{\mu I}$ (the standard isotropic elastic constitutive tensor, where $\lambda$ and $\mu$ are Lame's parameters and $\mathbf{1}$ and $\mathbf{I}$ are, respectively, the unit second- and fourthorder tensors) after some algebraic manipulations it turns out that

$$
a=\frac{1}{2(1-v)} \frac{1}{G}, \quad b=\frac{1}{G},
$$

where $v$ is Poisson's ratio and $G=\mu$ the shear modulus.

Condition (2) allows to define

$$
\mathbf{S} \stackrel{\text { def }}{=} \operatorname{sign}(\mathbf{P}) \sqrt{\mathbf{P} \cdot \mathbf{R}}=\operatorname{sign}(\mathbf{R}) \sqrt{\mathbf{R} \cdot \mathbf{P}}
$$

as a symmetric second-order tensor with real eigenvalues $s_{1}, s_{2}, s_{3}(s_{i}=\overbrace{\operatorname{sign}\left(p_{i}\right) \sqrt{p_{i} r_{i}}}^{\text {no sum }})$ and orthonormal eigenvectors $\hat{\mathrm{S}}_{1}, \hat{\mathrm{s}}_{2}, \hat{\mathrm{S}}_{3}$. For the associative case $(\mathbf{P}=\mathbf{R})$ it turns out that $\mathbf{S}=\mathbf{P}=\mathbf{R}$.

A variety of standard continuum dissipative constitutive models can be inserted in the previous class. As a matter of example in the following table values of the above entities for two families (elasto-plastic and damage [21]) of isotropic constitutive models are given:

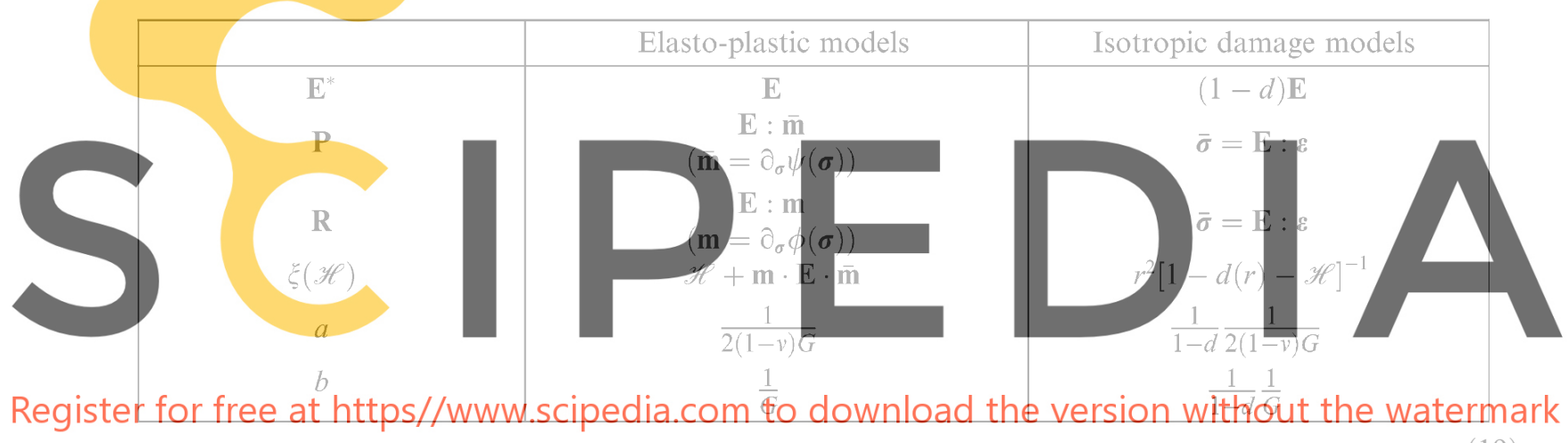

where $\phi$ and $\psi$ are, respectively, the yield and the plastic potential functions in plasticity models and $d(r) \in[0,1]$ is the damage variable in terms of the internal variable $r$.

\subsection{Geometric and closed form solutions}

For the class of the tangent constitutive operators defined in Section 3.1 it can be shown, after some algebraic manipulation, that Eq. (12) is equivalent to [35]:

$$
\begin{aligned}
& \xi(\mathscr{H})-\mathbf{n} \cdot \mathbf{P} \cdot \mathbf{Q}^{*^{-1}} \cdot \mathbf{R} \cdot \mathbf{n}=\boldsymbol{\xi}(\mathscr{H})-[b \mathbf{n} \cdot \mathbf{P} \cdot \mathbf{R} \cdot \mathbf{n}-a(\mathbf{n} \cdot \mathbf{P} \cdot \mathbf{n})(\mathbf{n} \cdot \mathbf{R} \cdot \mathbf{n})]=0 \\
& \quad \Rightarrow \xi(\mathscr{H})=b(\mathbf{n} \cdot \mathbf{P} \cdot \mathbf{R} \cdot \mathbf{n})-a(\mathbf{n} \cdot \mathbf{P} \cdot \mathbf{n})(\mathbf{n} \cdot \mathbf{R} \cdot \mathbf{n}),
\end{aligned}
$$

where Eqs. (11), (14) and (15) have been considered. In view of condition (3), the maximum value of $\mathscr{H} \equiv \mathscr{H}^{\text {crit }}$ in Eq. (13b) will correspond to the maximum value of $\xi \equiv \xi^{\text {crit }}$ in Eq. (20b). Now, let us consider the following statements:

$$
\mathbf{n} \cdot(\mathbf{P} \cdot \mathbf{R}) \cdot \mathbf{n}=\mathbf{n} \cdot \underbrace{(\mathbf{P} \cdot \mathbf{R})}_{\mathbf{S}^{2}=\mathbf{S} \cdot \mathbf{S}} \cdot \mathbf{n}=(\mathbf{n} \cdot \mathbf{S}) \cdot(\mathbf{S} \cdot \mathbf{n}),
$$

$$
(\mathbf{n} \cdot \mathbf{P} \cdot \mathbf{n})(\mathbf{n} \cdot \mathbf{R} \cdot \mathbf{n}) \cong(\mathbf{n} \cdot \mathbf{S} \cdot \mathbf{n})^{2}
$$


where Eq. (18) has been considered. It can be shown that the right- and left-hand-side terms of statement (21b) differ as the arithmetic and geometrical averages of couples $\left[\left|p_{i} r_{j}\right|,\left|p_{j} r_{i}\right|\right](i \neq j)$ do (see Appendix A). Now substituting Eqs. (21) into Eq. (20b) reads

$$
\begin{aligned}
& \xi(\mathscr{H})=b(\mathbf{n} \cdot \mathbf{P} \cdot \mathbf{R} \cdot \mathbf{n})-a(\mathbf{n} \cdot \mathbf{P} \cdot \mathbf{n})(\mathbf{n} \cdot \mathbf{R} \cdot \mathbf{n}) \cong \underbrace{b(\mathbf{n} \cdot \mathbf{S}) \cdot(\mathbf{S} \cdot \mathbf{n})-a(\mathbf{n} \cdot \mathbf{S} \cdot \mathbf{n})^{2}}_{\mathrm{Z}(\mathbf{n})}, \\
& \mathbf{Z}(\mathbf{n}) \stackrel{\text { def }}{=} b(\mathbf{n} \cdot \mathbf{S}) \cdot(\mathbf{S} \cdot \mathbf{n})-a(\mathbf{n} \cdot \mathbf{S} \cdot \mathbf{n})^{2} .
\end{aligned}
$$

In view of Eqs. (22) one can state that

$$
\begin{aligned}
& \mathbf{n}^{\text {crit }}=\underset{|\mathbf{n}|=1}{\arg }[\max Z(\mathbf{n})], \\
& \xi^{\text {crit }}\left(\mathscr{H}^{\text {crit }}\right)=Z\left(n^{\text {crit }}\right)
\end{aligned}
$$

provides an approximation for the solution $\mathscr{H}^{\text {crit }}$ in the original equation (13).

Remark 2. For the associative case ( $\mathbf{P}=\mathbf{R}=\mathbf{S})$ Eq. (22a) becomes an equality and solutions of problems (23) and (13) coincide (see Appendix A). In this case, the derivations below and the obtained values for $\mathscr{H}^{\text {crit }}$ and $\mathbf{n}^{\text {crit }}$, in Eqs. (27)-(29), are exact.
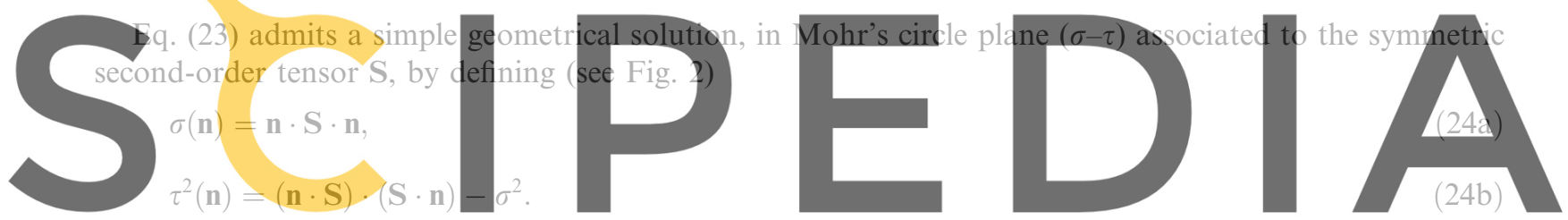

In that plane, Eq. (22b) reads in terms of the $(\sigma-\tau)$ coordinates:

Register for free at https//www.scipedia.com to down load the version without the watermark

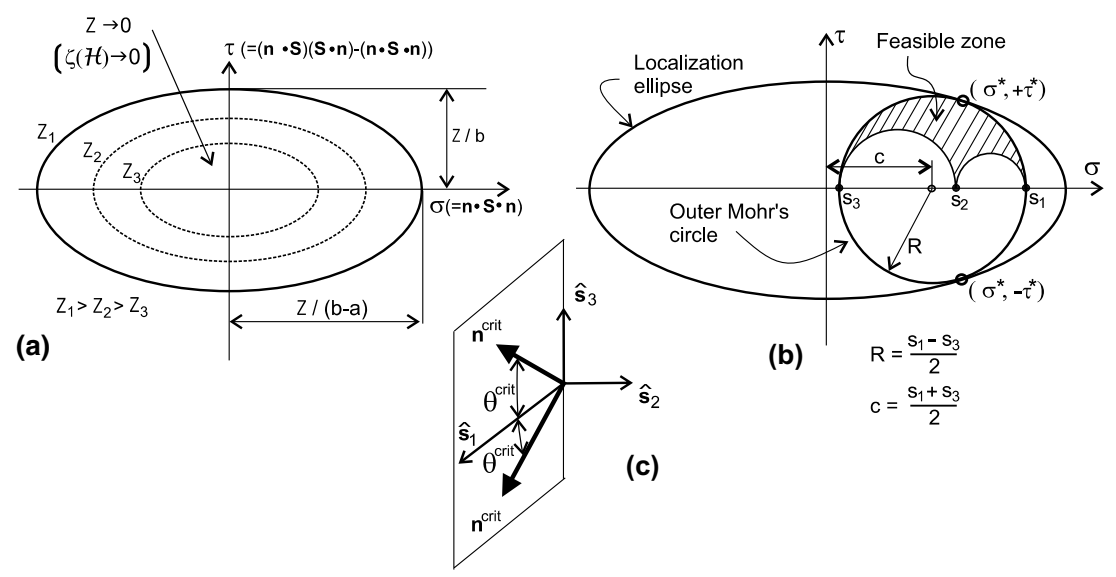

Fig. 2. Closed form material bifurcation solution: (a) localization ellipses in Mohr's space; (b) geometric solution; (c) direction of $\mathbf{n}^{\text {crit }}$ in the space of eigenvectors of $\mathbf{S}$. 
which can be rewritten as

$$
\frac{\sigma^{2}}{\frac{\mathrm{Z}}{b-a}}+\frac{\tau^{2}}{\frac{\mathrm{Z}}{b}}=1
$$

Eq. (26) defines in Mohr's plane an ellipse featuring the following properties (see Fig. 2a):

- The center of the ellipse is $(0,0)$.

- The ellipse semi-axes are $\frac{\mathrm{Z}}{b-a}$ and $\frac{\mathrm{Z}}{b}$. Therefore, the aspect ratio (shape) of the ellipse, defined as the ratio of both semi-axes, is $\frac{b}{b-a}$ (depending only on the material properties $a$ and $b$ ) and therefore it is independent of Z.

- The size of the ellipse (defined by the semi-axes values) is proportional to the value of $Z$.

Consequently, decreasing values of $Z$ will define concentric smaller and smaller ellipses as it is shown in Fig. 2a. Solutions of the bifurcation problem shall lie in the intersection of the ellipse with the feasible zone inside Mohr's circles area. Solutions corresponding to the highest value of Z and, therefore, to the highest (critical) values of $\xi^{\text {crit }}$ and $\mathscr{H}^{\text {crit }}$ in Eq. (23b) will be obtained for the tangent ellipse to the outer Mohr's' circle in Fig. 2b. After some algebraic manipulations, $\mathbf{n}^{\text {crit }}$ can be solved as (see Appendix B)

$$
\mathbf{n}^{\text {crit }}=\cos \theta^{\text {crit }} \hat{\mathbf{s}}_{1}+\sin \theta^{\text {crit }} \hat{\mathbf{s}}_{3} \text {, }
$$

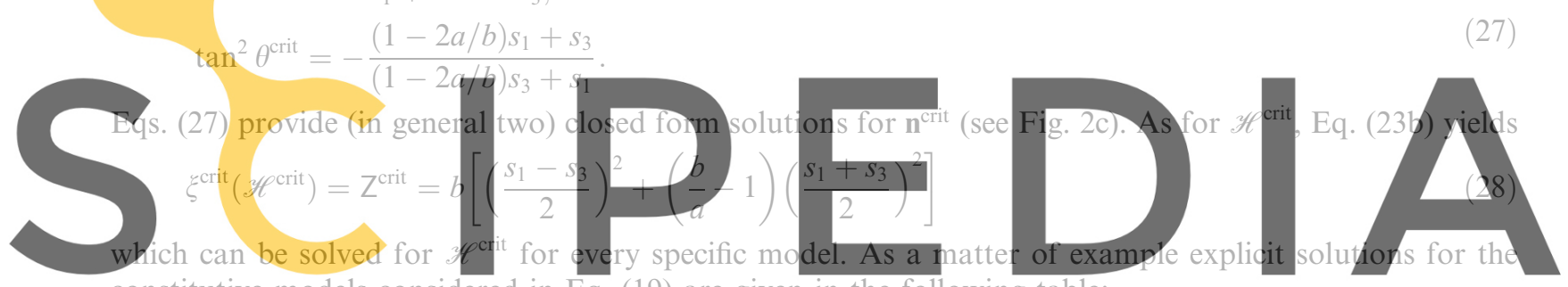

constitutive models considered in Eq. (19) are given in the following table:

Register for free at https//www.scipedia.com to download the version without the watermark

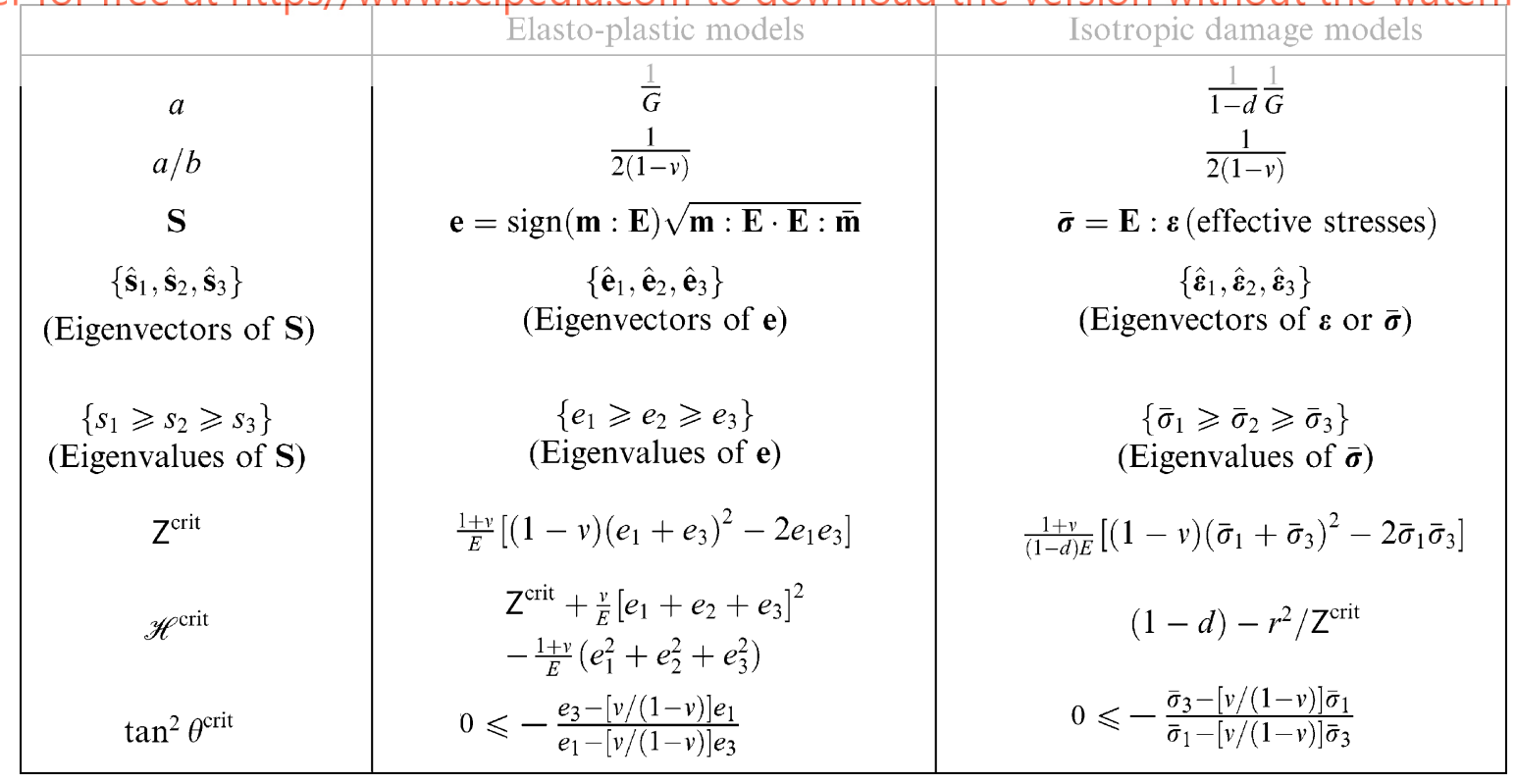




\section{Finite element modelling}

Computational modelling of strong discontinuities has been object of increasing development during the last years $[1,7,13,15,16,25,32]$. In the finite element context, specific elements to capture displacement jumps by means of enriching deformation modes seem to be the best available option. This technology consists essentially of adding, to standard continuum finite elements, enriching deformation modes, controlled by additional degrees of freedom. The resulting elements capture displacement discontinuities placed anywhere inside them and have been termed finite elements with embedded discontinuities. Then, depending on the support of those additional displacement modes they can be split up into two families:

1. Nodal enrichment (see for instance [13,17,34]). The support of the enriching discontinuous mode is (in most cases) the same than for the nodal shape functions of the underlying element (see Fig. 3a). The regular nodes of the enriched set of elements are increased with additional degrees of freedom (two per node in 2D cases, three node in 3D cases) whose interpolation provides a, varying inside the element, discontinuous displacement field.

2. Elemental enrichment (see for instance $[3,10,18,20,25,29]$ ). The support of the enriching discontinuity mode is elemental (see Fig. 3b) and, in consequence, the additional internal discontinuous degrees of
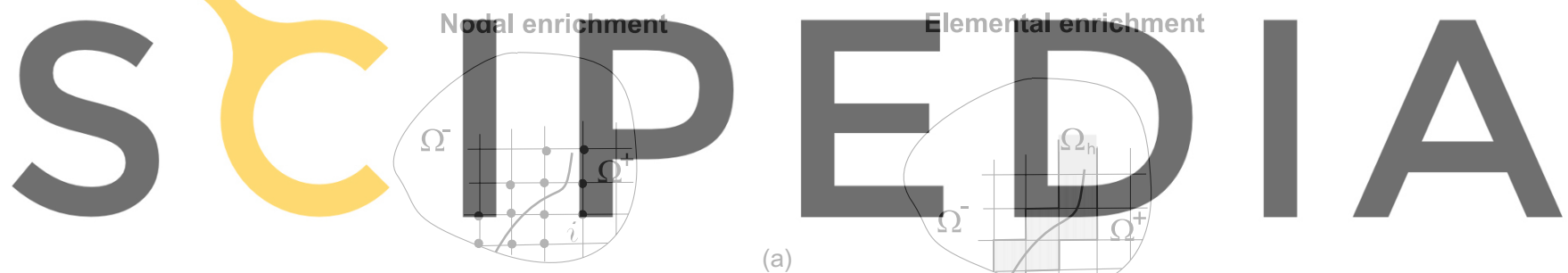

Register for free at https//www.scipedia.com to download the version without the watermark

Enriching modes

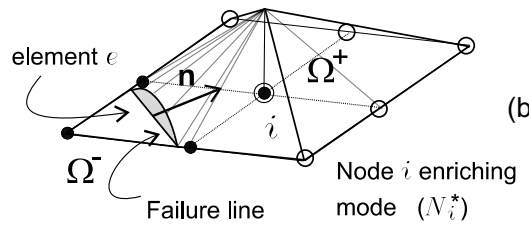

(b)
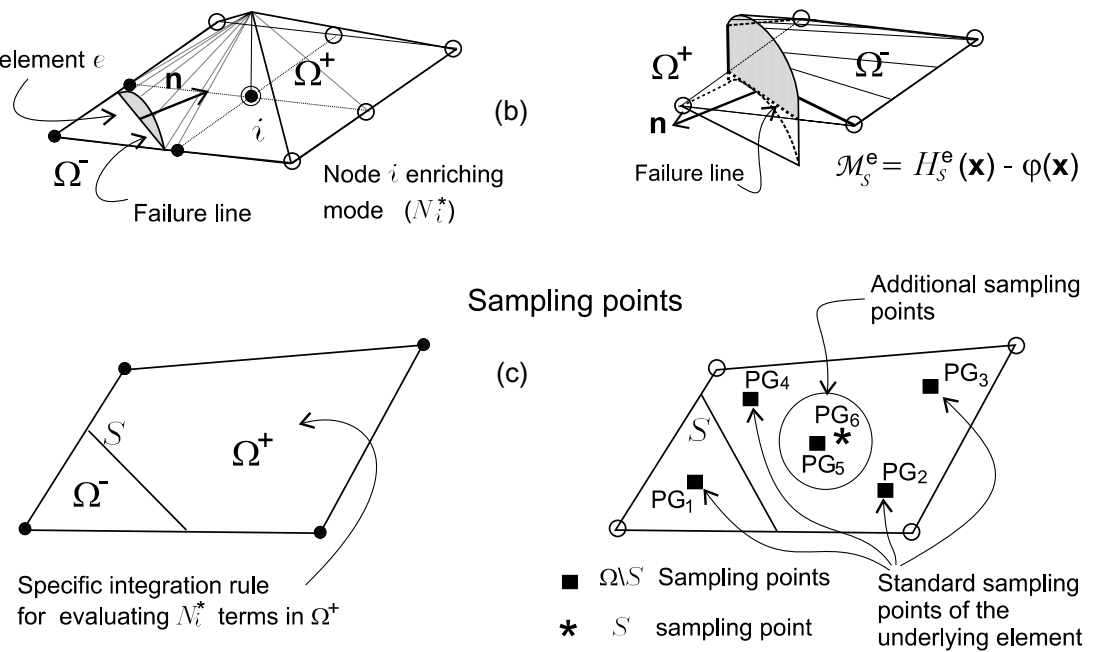

Fig. 3. Finite elements with embedded discontinuities: nodal vs. elemental enriching techniques; (a) enriched nodes and elements; (b) enhanced shape functions and support; (c) sampling points for numerical integration. 
freedom (two per element in 2D cases and three per element in 3D cases) can be condensed at the elemental level. The displacement jump is element-wise constant.

In the following sections those finite elements are described.

\subsection{Boundary value problem}

The boundary value continuum problem for a strong discontinuity propagating in a body $\Omega$ along a failure surface $\mathscr{S}$ of normal $\mathbf{n}$, that splits $\Omega$ into the domains $\Omega^{+}$and $\Omega^{-}$(see Fig. 4), reads (in rate form):

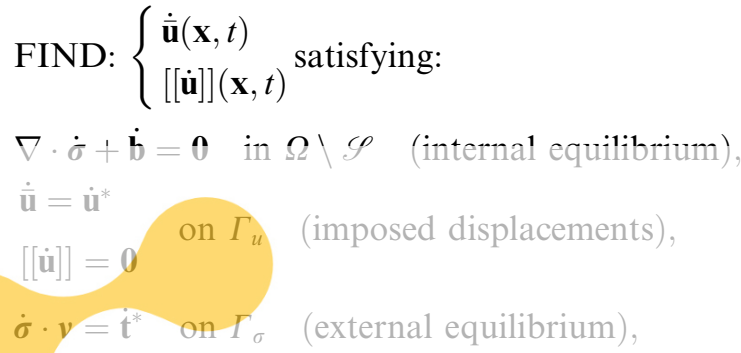

$\dot{\boldsymbol{\sigma}} \cdot \boldsymbol{y}=\dot{\mathbf{t}}^{*} \quad$ on $\Gamma_{\sigma} \quad($ external equilibrium $)$,

$\underbrace{\dot{\boldsymbol{\sigma}}_{\Omega^{-}} \cdot \mathbf{n}-\dot{\boldsymbol{\sigma}}_{\Omega^{+}} \cdot \mathbf{n}}=\mathbf{0} \quad$ on $\mathscr{S} \quad$ (outer traction continuity),
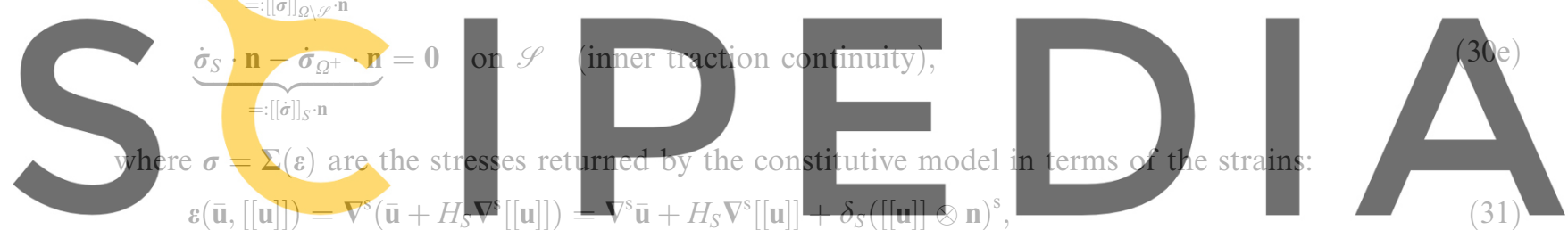

Regi $\mathbf{b}\left(\mathbf{x}_{\mathrm{f}} t\right)$ are the body forces, $\mathbf{u}^{*}$ are the prescribed displacements and $\mathrm{t}^{*}$ the prescribed.traction at the external Register for free at https and $\sigma_{S}$ stand for the stresses in $\Omega^{+}, \Omega^{-}$and $\mathscr{S}$, respectively.

\subsection{Nodal enrichment: symmetric formulation}

Let us consider the following variational problem:

GIVEN:

$$
\begin{aligned}
& \mathscr{V}_{\eta}:=\left\{\boldsymbol{\eta}=\overline{\boldsymbol{\eta}}+H_{S} \tilde{\boldsymbol{\eta}}^{0} ; \overline{\boldsymbol{\eta}},\left.\tilde{\boldsymbol{\eta}}^{0} \in\left[H^{1}(\Omega)\right]^{n_{\operatorname{dim}}} \tilde{\boldsymbol{\eta}}^{0}\right|_{\Gamma_{u}}=\left.\mathbf{0} \overline{\boldsymbol{\eta}}\right|_{\Gamma_{u}}=\dot{\mathbf{u}}^{*}\right\}, \\
& \mathscr{V}_{\eta}^{0}:=\left\{\boldsymbol{\eta}^{0}=\overline{\boldsymbol{\eta}}^{0}+H_{S} \tilde{\boldsymbol{\eta}}^{0} ; \overline{\boldsymbol{\eta}}^{0},\left.\tilde{\boldsymbol{\eta}}^{0} \in\left[H^{1}(\Omega)\right]^{n_{\mathrm{dim}}} \tilde{\boldsymbol{\eta}}^{0}\right|_{\Gamma_{u}}=\left.\mathbf{0} \overline{\boldsymbol{\eta}}^{0}\right|_{\Gamma_{u}}=\mathbf{0}\right\} .
\end{aligned}
$$
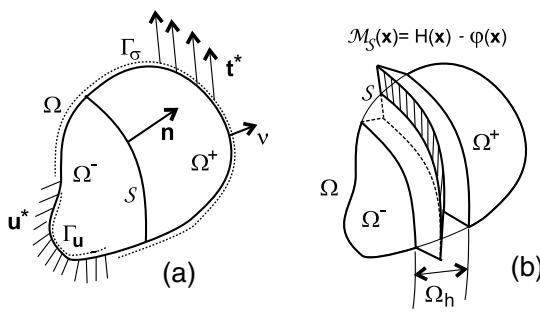

Fig. 4. Failure surface $\mathscr{S}$ propagating in a body $\Omega$ : (a) boundary value problem; (b) unit jump function $\mathscr{M}_{S}$. 
FIND:

$$
\dot{\mathbf{u}} \in \mathscr{V}_{\eta} ; \quad \dot{\mathbf{u}}=\dot{\overline{\mathbf{u}}}+H_{S}[[\dot{\mathbf{u}}]]
$$

\section{SUCH THAT:}

$$
\int_{\Omega} \nabla^{S} \boldsymbol{\eta}^{0}: \dot{\boldsymbol{\sigma}} \mathrm{d} \Omega-\left[\int_{\Omega} \boldsymbol{\eta}^{0} \cdot \dot{\mathbf{b}} \mathrm{d} \Omega+\int_{\Gamma_{\sigma}} \boldsymbol{\eta}^{0} \cdot \dot{\mathbf{t}}^{*} \mathrm{~d} \Gamma\right]=0 \quad \forall \boldsymbol{\eta}^{0} \in \mathscr{V}_{\eta}^{0},
$$

where $n_{\mathrm{dim}}$ stands for the dimension of the problem $\left(n_{\mathrm{dim}}=2\right.$ for $2 \mathrm{D}$ and $n_{\mathrm{dim}}=3$ for $\left.3 \mathrm{D}\right)$. It can be readily shown that the Euler-Lagrange equations of variational equation (34) are the ones defining the BVP (30). Eq. (34) is the starting point for the nodal-based enriching formulation. The spatial discretization of the displacement fields $\dot{\mathbf{u}}$ and $\boldsymbol{\eta}$ in Eqs. (32) and (33) reads:

$$
\begin{aligned}
& \dot{\mathbf{u}}^{h}(\mathbf{x}, t)=\sum_{i=1}^{i=n_{\text {node }}} N_{i}(\mathbf{x}) \dot{\mathbf{d}}_{i}(t)+\sum_{i=1}^{i=n_{\text {enr }}} \underbrace{N_{i} H_{S}(\mathbf{x})}_{N_{i}^{*}} \dot{\boldsymbol{\beta}}_{i}(t)=\underbrace{\sum_{i=1}^{i=n_{\text {node }}} N_{i} \dot{\mathbf{d}}_{i}}_{[\mathbf{N}]\{\mathbf{d}\}}+\underbrace{\sum_{i=1}^{i=n_{\text {enr }}} N_{i}^{*} \dot{\boldsymbol{\beta}}_{i}}_{\left[\mathbf{N}^{*}\right]\{\beta\}} \\
& \boldsymbol{\eta}^{h^{0}}(\mathbf{x}, t)=\sum_{i=1}^{i=n_{\text {node }}} N_{i} \overline{\boldsymbol{\eta}}_{i}^{0}+\sum_{i=1}^{i=n_{\text {enr }}} N_{i}^{*} \tilde{\boldsymbol{\eta}}_{i}^{0}, \\
& \left\{\dot{\mathbf{u}}^{h}\right\}=[\mathbf{N}]\{\dot{\mathbf{d}}\}+\left[\mathbf{N}^{*}\right]\{\dot{\beta}\} ; \quad N_{i}^{*}(\mathbf{x})=N_{i}(\mathbf{x}) H_{S}(\mathbf{x}), \\
& \{\dot{\mathbf{d}}\}=\left[\dot{\mathbf{d}}_{1}, \ldots, \dot{\mathbf{d}}_{n_{\text {node }}}\right]^{\mathrm{T}} ; \quad\{\dot{\boldsymbol{\beta}}\}=\left[\dot{\boldsymbol{\beta}}_{1}, \ldots, \dot{\boldsymbol{\beta}}_{n_{\text {enr }}}\right]^{\mathrm{T}},
\end{aligned}
$$

where $n_{\text {node }}$ stands for the number of nodes of the finite element mesh, $N_{i}(\mathbf{x})$ are the shape functions of the parent, standard, element and $\mathbf{d}_{i}$ are the regular nodal displacements. Also in Eq. (35) $\boldsymbol{\beta}_{i}$ are additional nodal degrees of freedom such that the enriching modes $N_{i}^{*} \dot{\boldsymbol{\beta}}_{i}$ capture the displacements jumps in an interelemental continuous way (see Fig. $3 \mathrm{~b}$ ) and $n_{\mathrm{enr}}$ is the number of nodal points where the enrichment is

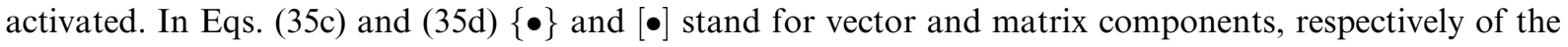
entity $(\bullet)$.

In order to fulfill condition $\left.\tilde{\boldsymbol{\eta}}^{0}\right|_{\Gamma_{u}}=\mathbf{0}$ in Eqs. (32) the enriching degrees of freedom $\boldsymbol{\beta}_{i}$ have to be prescribed to zero at those nodes whose support (surrounding elements) contain points of $\Gamma_{u}$. As for the discretized strain field $\dot{\boldsymbol{\varepsilon}}^{h}$ it yields from Eq. (35a):

$$
\dot{\boldsymbol{\varepsilon}}^{h}(\mathbf{x}, t)=\nabla^{\mathrm{s}} \dot{\mathbf{u}}(\mathbf{x}, t)=\underbrace{\sum_{i=1}^{i=n_{\text {node }}}\left(\boldsymbol{\nabla} N_{i} \otimes \dot{\mathbf{d}}_{i}\right)^{S}}_{[\mathbf{B}]\{\dot{\mathbf{d}}\}}+\underbrace{\sum_{i=1}^{i=n_{\mathrm{enr}}}\left\{N_{i} \delta_{S}^{k}\left(\mathbf{n} \otimes \dot{\boldsymbol{\beta}}_{i}\right)^{S}+H_{S}\left(\boldsymbol{\nabla} N_{i} \otimes \dot{\boldsymbol{\beta}}_{i}\right)^{S}\right\}}_{[\mathbf{G}]\{\dot{\boldsymbol{\beta}}\}},
$$

where $\delta_{S}^{k}$ stand for the $k$-regularized Dirac's delta function placed at the failure interface $S$ (see Eq. (5)). In matrix (Voigt's) notation, Eq. (35) reads:

$$
\left\{\dot{\boldsymbol{\varepsilon}}^{h}\right\}=[\mathbf{B}]\{\dot{\mathbf{d}}\}+[\mathbf{G}]\{\dot{\boldsymbol{\beta}}\},
$$

where $[\mathbf{B}]$ stands for the standard deformation matrix [36] and the structure of the additional matrix $[\mathbf{G}]$ comes out directly from Eq. (36). Substitution of Eq. (37) into the discrete form of the variational equation (34) leads to the linearized discretized problem:

$$
\left[\begin{array}{cc}
\int_{\Omega}[\mathbf{B}]^{\mathrm{T}}[\mathscr{D}][\mathbf{B}] \mathrm{d} \Omega & \int_{\Omega}[\mathbf{B}]^{\mathrm{T}}[\mathscr{D}][\mathbf{G}] \mathrm{d} \Omega \\
\int_{\Omega}[\mathbf{G}]^{\mathrm{T}}[\mathscr{D}][\mathbf{B}] \mathrm{d} \Omega & \int_{\Omega}[\mathbf{G}]^{\mathrm{T}}[\mathscr{D}][\mathbf{G}] \mathrm{d} \Omega
\end{array}\right]\left\{\begin{array}{c}
\dot{\mathbf{d}} \\
\dot{\boldsymbol{\beta}}
\end{array}\right\}=\left\{\begin{array}{l}
\dot{\mathbf{f}}_{\mathbf{d}} \\
\dot{\mathbf{f}}_{\boldsymbol{\beta}}
\end{array}\right\}
$$




$$
\left\{\begin{array}{c}
\dot{\mathbf{f}}_{\mathrm{d}} \\
\dot{\mathbf{f}}_{\boldsymbol{\beta}}
\end{array}\right\}=\left\{\begin{array}{c}
\int_{\Omega}[\mathbf{N}]^{\mathrm{T}}\{\dot{\mathbf{b}}\} \mathrm{d} \Omega+\int_{\Gamma_{\sigma}}[\mathbf{N}]^{\mathrm{T}}\left\{\dot{\mathbf{t}}^{*}\right\} \mathrm{d} \Gamma \\
\int_{\Omega}\left[\mathbf{N}^{*}\right]^{\mathrm{T}}\{\dot{\mathbf{b}}\} \mathrm{d} \Omega+\int_{\Gamma_{\sigma}}\left[\mathbf{N}^{*}\right]^{\mathrm{T}}\left\{\dot{\mathbf{t}}^{*}\right\} \mathrm{d} \Gamma
\end{array}\right\},
$$

where $[\mathscr{D}]$ is the matrix counterpart of the tangent constitutive tensor $\mathbf{E}^{\text {tang }}$ in Eq. (14) ruling the incremental constitutive equation in Voigt's notation: $\left\{\dot{\boldsymbol{\sigma}}^{h}\right\}=[\mathscr{D}]\left\{\dot{\boldsymbol{\varepsilon}}^{h}\right\}$.

Remark 3. The appearance of the regularized Dirac's delta function in Eq. (36) requires additional sampling points inside the elements to evaluate its contribution to Eqs. (38) (see [20] for more details). Also terms $N_{i} H_{S}$ and $H_{S} \nabla^{\mathrm{s}}\left(N_{i}\right)$ in Eqs. (35) and (36) are discontinuous inside the element, which requires specific integration rules at every side $\Omega_{\mathrm{e}}^{+}$and $\Omega_{\mathrm{e}}^{+}$of any enriched element, and knowledge of the exact position of the failure surface inside the elements. The algorithms devised to determine that position are termed tracking algorithms and some alternatives on this subject are presented in [26].

\subsection{Elemental enrichment: non-symmetric formulation}

Let us consider the domain $\Omega$ crossed by the failure surface and a subdomain $\Omega_{h}$ containing $S$ and such that $\Omega_{h} \cap \Gamma_{u}=\{\emptyset\}$. Let us also define a unit jump function $\mathscr{M}_{S}$ (see Fig. $4 \mathrm{~b}$ ) as

$$
\mathscr{M}_{S}(\mathbf{x})=H_{S}(\mathbf{x})-\varphi(\mathbf{x}), \quad \varphi(\mathbf{x}) \in H^{1}\left(\Omega_{h}\right):= \begin{cases}\varphi(\mathbf{x})=0 & \forall x \in \Omega^{-} \backslash \Omega_{h} \\ \varphi(\mathbf{x})=1 & \forall x \in \Omega^{+} \backslash \Omega_{h}\end{cases}
$$

exhibiting a unit jump across the failure surface $S$. Let us now consider the following variational problem:

GIVEN:

$$
\begin{aligned}
& \mathscr{V}_{\eta}:=\left\{\boldsymbol{\eta}=\hat{\boldsymbol{\eta}}+\mathscr{M}_{S} \tilde{\boldsymbol{\eta}} ;\left.\hat{\boldsymbol{\eta}} \in\left[H^{1}(\Omega)\right]^{n_{\mathrm{dim}}} \tilde{\boldsymbol{\eta}} \in L_{2}(S) \hat{\boldsymbol{\eta}}\right|_{\Gamma_{u}}=\dot{\mathbf{u}}^{*}\right\}, \\
& \mathscr{V}_{\hat{\eta}}^{0}:=\left\{\left.\hat{\boldsymbol{\eta}}^{0} \in\left[H^{1}(\Omega)\right]^{n_{\mathrm{dim}}} \hat{\boldsymbol{\eta}}^{0}\right|_{\Gamma_{u}}=\mathbf{0}\right\} .
\end{aligned}
$$

FIND:

$$
\dot{\mathbf{u}} \in \mathscr{V}_{\eta} ; \quad \dot{u}=\dot{\overline{\mathbf{u}}}+\mathscr{M}_{S}[[\dot{\mathbf{u}}]]
$$

SUCH THAT:

$$
\int_{\Omega \backslash S} \nabla^{S} \hat{\boldsymbol{\eta}}^{0}: \dot{\boldsymbol{\sigma}} \mathrm{d} \Omega-\left[\int_{\Omega \backslash S} \hat{\boldsymbol{\eta}}^{0} \cdot \dot{\mathbf{b}} \mathrm{d} \Omega+\int_{\Gamma_{\sigma}} \hat{\boldsymbol{\eta}}^{0} \cdot \dot{\mathbf{t}}^{*} \mathrm{~d} \Gamma\right]=0 \quad \forall \hat{\boldsymbol{\eta}}^{0} \in \mathscr{V}_{\hat{\eta}}^{0}
$$

Remark 4. It can be readily checked that any element of the solution space $\mathscr{V}_{\eta}$ in Eq. (40a) can be cast into the original format of Eq. (1a).

Due to the difference between the functional spaces $\mathscr{V}_{\eta}^{0}$ and $\mathscr{V}_{\bar{\eta}}^{0}$ in Eqs. (32b) and (40b) now the EulerLagrange equations of the variational form (42) are only Eqs. (30a)-(30d). Therefore, the inner traction continuity equation (30e) has to be imposed independently of the variational statement (42). This can be done in weak form through:

$$
\int_{S} \tilde{\boldsymbol{\eta}} \cdot\left(\dot{\boldsymbol{\sigma}}_{S}-\dot{\boldsymbol{\sigma}}_{\Omega \backslash S}\right) \cdot \mathbf{n} \mathrm{d} S=0 \quad \forall \tilde{\boldsymbol{\eta}} \in L_{2}(S) .
$$


The discrete version of $\dot{\mathbf{u}}(\mathbf{x}, t), \hat{\eta}^{0}(\mathbf{x}, t)$ and $\hat{\boldsymbol{\eta}}(\mathbf{x}, t)$ in Eqs. (40) and (41) reads:

$$
\begin{aligned}
& \dot{\mathbf{u}}^{h}(\mathbf{x}, t)=\underbrace{\sum_{i=1}^{1=n_{\text {node }}} N_{i}(\mathbf{x}) \dot{\mathbf{d}}_{i}(t)}_{[\mathbf{N}]\{\mathbf{d}\}}+\underbrace{\sum_{e=1}^{e=n_{\mathrm{enr}}} \mathscr{M}_{S}^{e}(\mathbf{x}) \dot{\boldsymbol{\beta}}^{e}(t),}_{[\mathcal{M}]\{\dot{\boldsymbol{\beta}}\}} \\
& \boldsymbol{\eta}^{h^{0}}(\mathbf{x}, t)=\underbrace{\sum_{i=1}^{1=n_{\text {node }}} N_{i}(\mathbf{x}) \hat{\eta}_{i}(t)}_{\hat{\boldsymbol{\eta}}^{h}(\mathbf{x}, t)}+\sum_{e=1}^{e=n_{\mathrm{enr}}} \mathscr{M}_{S}^{e}(\mathbf{x}) \tilde{\boldsymbol{\eta}}^{e}(t), \\
& \left\{\dot{\mathbf{u}}^{h}\right\}=[\mathbf{N}]\{\dot{\mathbf{d}}\}+[\mathscr{M}]\{\dot{\boldsymbol{\beta}}\} ; \quad \mathscr{M}_{S}^{e}=H_{S}^{e}(\mathbf{x})-\boldsymbol{\varphi}^{e}, \\
& \{\dot{\mathbf{d}}\}=\left[\dot{\mathbf{d}}_{1}, \ldots, \dot{\mathbf{d}}_{n_{\text {node }}}\right]^{\mathrm{T}} ; \quad\{\dot{\boldsymbol{\beta}}\}=\left[\dot{\boldsymbol{\beta}}^{1}, \ldots, \dot{\boldsymbol{\beta}}^{n_{\mathrm{enr}}}\right]^{\mathrm{T}},
\end{aligned}
$$

where $n_{\mathrm{enr}}$ is the number of enriched elements and $\mathscr{M}_{S}^{e}(\mathbf{x})$ is the so-called elemental unit jump function:

$$
\mathscr{M}_{S}^{e}(\mathbf{x})=H_{S}(\mathbf{x})-\varphi^{e}(\mathbf{x}), \quad \varphi^{e}(\mathbf{x}) \in H^{1}\left(\Omega_{h}^{e}\right):= \begin{cases}\varphi^{e}(\mathbf{x})=0 & \forall x \in \Omega^{-} \backslash \Omega_{h}^{e} \\ \varphi^{e}(\mathbf{x})=1 & \forall x \in \Omega^{+} \backslash \Omega_{h}^{e}\end{cases}
$$

with elemental support as shown in Fig. $3 b$. The term $\mathscr{M}_{S}^{e}(\mathbf{x}) \dot{\boldsymbol{\beta}}^{e}(t)$ provides the enriching elemental mode where $\boldsymbol{\beta}^{e}$ stands for the elemental displacement jump. As for the discretized strain field $\dot{\boldsymbol{\varepsilon}}^{h}$ it yields from Eqs. (44a) and (45):

$$
\dot{\boldsymbol{\varepsilon}}^{h}(\mathbf{x}, t)=\nabla^{\mathrm{s}} \dot{\mathbf{u}}^{h}(\mathbf{x}, t)=\underbrace{\sum_{i=1}^{i=n_{\text {node }}}\left(\boldsymbol{\nabla} N_{i} \otimes \dot{\mathbf{d}}_{i}\right)^{S}}_{[\mathbf{B}]\{\dot{\mathbf{d}}\}}+\underbrace{\sum_{e=1}^{e=n_{\text {enr }}}\left[\delta_{S}^{k}\left(\mathbf{n} \otimes \dot{\boldsymbol{\beta}}^{e}\right)^{S}-\left(\nabla \boldsymbol{\varphi}^{e} \otimes \dot{\boldsymbol{\beta}}^{e}\right)^{S}\right]}_{[\mathbf{G}]\{\dot{\beta}\}},
$$

where $\delta_{S}^{k}$ stands for the $k$-regularized Dirac's delta function shifted to the failure surface $S$ (see Eq. (5)). In matrix notation, Eq. (46) reads

$$
\left\{\dot{\boldsymbol{\varepsilon}}^{h}\right\}=[\mathbf{B}]\{\dot{\mathbf{d}}\}+[\mathbf{G}]\{\dot{\boldsymbol{\beta}}\},
$$

where the format of matrices $[\mathbf{B}]$ and $[\mathbf{G}]$ comes readily out from Eq. (46).

As for the inner traction continuity enforced by Eq. (43) $\dot{\boldsymbol{\sigma}}_{S} \cdot \mathbf{n}$ and $\dot{\boldsymbol{\sigma}}_{\Omega \backslash S} \cdot \mathbf{n}$ can be approximated by its average values inside every element, i.e.:

$$
\begin{aligned}
& \left.\dot{\boldsymbol{\sigma}}_{S} \cdot \mathbf{n}\right|_{\mathbf{x} \in S^{e}} \approx \frac{1}{l^{e}} \int_{S^{e}} \dot{\boldsymbol{\sigma}}\left(\boldsymbol{\varepsilon}_{S}\right) \cdot \mathbf{n}^{e} \mathrm{~d} S=\dot{\boldsymbol{\sigma}}_{S}\left(\mathbf{x}_{S^{e}}^{*}\right) \cdot \mathbf{n}^{e}, \quad \mathbf{x}_{S^{e}}^{*} \in S^{e}, \\
& \left.\dot{\boldsymbol{\sigma}}_{\Omega \backslash S} \cdot \mathbf{n}\right|_{\mathbf{x} \in \Omega^{e}} \approx \frac{1}{V^{e}} \int_{\Omega^{e}} \dot{\boldsymbol{\sigma}}\left(\boldsymbol{\varepsilon}_{\Omega \backslash S}\right) \cdot \mathbf{n}^{e} \mathrm{~d} \Omega=\dot{\boldsymbol{\sigma}}_{\Omega \backslash S}\left(\mathbf{x}_{\Omega^{e}}^{*}\right) \cdot \mathbf{n}^{e}, \quad \mathbf{x}_{\Omega^{e}}^{*} \in \Omega^{e},
\end{aligned}
$$

where $V^{e}=$ measure $\left(\Omega^{e}\right), l^{e}=$ measure $\left(S^{e}\right)$ and $\mathbf{x}_{\Omega^{e}}^{*}$ and $\mathbf{x}_{S^{e}}^{*}$ are those points at the interior of $\Omega^{e}$ and $S^{e}$ where the mean values of $\dot{\boldsymbol{\sigma}}\left(\boldsymbol{\varepsilon}_{\Omega \backslash S}\right)$ and $\dot{\boldsymbol{\sigma}}\left(\boldsymbol{\varepsilon}_{S}\right)$, respectively, are reached. For practical purposes they are two additional sampling points sharing the same geometrical position, at the centroid of the element, see Fig. 3c.

In this context, the discretized version of $\tilde{\boldsymbol{\eta}}$ in Eq. (43) yields:

$$
\tilde{\boldsymbol{\eta}}^{h}(\mathbf{x}, t)=\underbrace{\sum_{e=1}^{e=n_{\mathrm{enr}}} \chi^{e}(\mathbf{x}) \tilde{\boldsymbol{\eta}}^{e}(t)}_{\left[\mathbf{G}^{*}\right]\{\tilde{\boldsymbol{\eta}}\}} ; \quad \chi^{e}(\mathbf{x})= \begin{cases}\zeta(\mathbf{x}) & \forall \mathbf{x} \in \Omega^{e}, \\ 0 & \forall \mathbf{x} \notin \Omega^{e},\end{cases}
$$




$$
\begin{aligned}
& \zeta(\mathbf{x})=l^{e}\left[\mu_{\mathbf{x}_{S^{e}}^{*}}(\mathbf{x}) / W_{\mathbf{x}_{S^{e}}^{*}}-\mu_{\mathbf{x}_{\Omega^{e}}^{*}}(\mathbf{x}) / W_{\mathbf{x}_{\Omega^{e}}^{*}}\right], \\
& \left\{\tilde{\boldsymbol{\eta}}^{h}\right\}=\left[\mathbf{G}^{*}\right]\{\tilde{\boldsymbol{\eta}}\} ; \quad\{\tilde{\boldsymbol{\eta}}\}=\left[\tilde{\boldsymbol{\eta}}^{1}, \ldots, \tilde{\boldsymbol{\eta}}^{n_{\mathrm{enr}}}\right]^{\mathrm{T}},
\end{aligned}
$$

where $\mu_{\mathbf{x}_{S^{e}}^{*}}(\mathbf{x})$ and $\mu_{\mathbf{x}_{\Omega^{e}}^{*}}(\mathbf{x})$ are collocation functions at the sampling points $\mathbf{x}_{S^{e}}^{*}$ and $\mathbf{x}_{\Omega^{e}}^{*}$, respectively $\left(\mu_{\mathbf{y}}(\mathbf{x})=\right.$ 1 for $\mathbf{x}=\mathbf{y}$ and $\mu_{\mathbf{y}}(\mathbf{x})=0$ otherwise) and $W_{\mathbf{x}_{S^{e}}^{*}}$ and $W_{\mathbf{x}^{e}}$ are the integration weights at the additional sampling points.

Substitution of Eqs. (46)-(48) into the discrete form of the integral equations (42) and (43) leads to the linearized problem:

$$
\begin{aligned}
& {\left[\begin{array}{cc}
\int_{\Omega}[\mathbf{B}]^{\mathrm{T}}[\mathscr{D}][\mathbf{B}] \mathrm{d} \Omega & \int_{\Omega}[\mathbf{B}]^{\mathrm{T}}[\mathscr{D}][\mathbf{G}] \mathrm{d} \Omega \\
\int_{\Omega}\left[\mathbf{G}^{*}\right]^{\mathrm{T}}[\mathscr{D}][\mathbf{B}] \mathrm{d} \Omega & \int_{\Omega}\left[\mathbf{G}^{*}\right]^{\mathrm{T}}[\mathscr{D}][\mathbf{G}] \mathrm{d} \Omega
\end{array}\right]\left\{\begin{array}{c}
\dot{\mathbf{d}} \\
\dot{\boldsymbol{\beta}}
\end{array}\right\}=\left\{\begin{array}{c}
\dot{\mathbf{f}}_{\mathbf{d}} \\
\mathbf{0}
\end{array}\right\},} \\
& \dot{\mathbf{f}}_{\mathbf{d}}=\int_{\Omega}[\mathbf{N}]^{\mathrm{T}}\{\dot{\mathbf{b}}\} \mathrm{d} \Omega+\int_{\Gamma_{\sigma}}[\mathbf{N}]^{\mathrm{T}}\left\{\dot{\mathbf{t}}^{*}\right\} \mathrm{d} S .
\end{aligned}
$$

Notice that, from their definition in Eqs. (46) and (49), matrices $[\mathbf{G}]$ and $\left[\mathbf{G}^{*}\right]$ are different and the resulting formulation is non-symmetric, as one should expect from the Petrov-Galerkin character of the original problem in Eqs. (40)-(43).

Remark 5. Determination of the elemental unit jump function in Eq. (45) (see Fig. 3a) requires, to some extent, knowledge of the position of the failure surface $S$ in every enriched element. Thus, like in the nodal enrichment case, this fact requires resorting to tracking algorithms for determination of that position (see Remark 3).

Remark 6. As for the integration rule, the weight of the additional sampling points in $\mathbf{x}_{\Omega^{e}}^{*}$ and $\mathbf{x}_{S^{e}}^{*}\left(W_{\mathbf{x}_{\Omega^{e}}^{*}}\right.$ and $W_{\mathbf{x}_{s e}^{*}}$, respectively) should be made very small and, thus, their contribution made negligible except for imposing Eq. (30e) through (43). Therefore, they can be incorporated to all elements, even to those nonenriched. In this sense, and unlike the nodal enrichment case in Section 4.2, distinction, in terms of the integration rule, between elements in and out the enriched domain is not necessary.

\section{Instability issues: loss of uniqueness}

It is a very well-known fact in failure mechanics that material softening is responsible for unstable structural behavior [4,5]. For purposes of numerical modelling of the postcritical structural behavior, some of those instabilities, like limit or turning points, depend on the load pattern, and the response can be easily traced by resorting to appropriate continuation methods [9]. More difficulties are found in presence of bifurcation points (loss of uniqueness) which require detection and continuation through appropriate branch-switching methods. This situation, which can appear even when a unique failure surface is modelled, worsens as multiple failure surfaces, interacting with each other and capable to arrest and activate, are tackled.

A possible way to get rid of the difficulties posed by bifurcation points in material instability simulations is to resort to unfolding techniques. Those techniques are based on introducing perturbations to unfold and smooth the different equilibrium paths emanating from a bifurcation point in such a way that the unique stable path remains continuous (see Fig. 5) and, therefore, it can be theoretically traced as an approximation to the physical equilibrium path. The success of such methodology relies on two facts: 


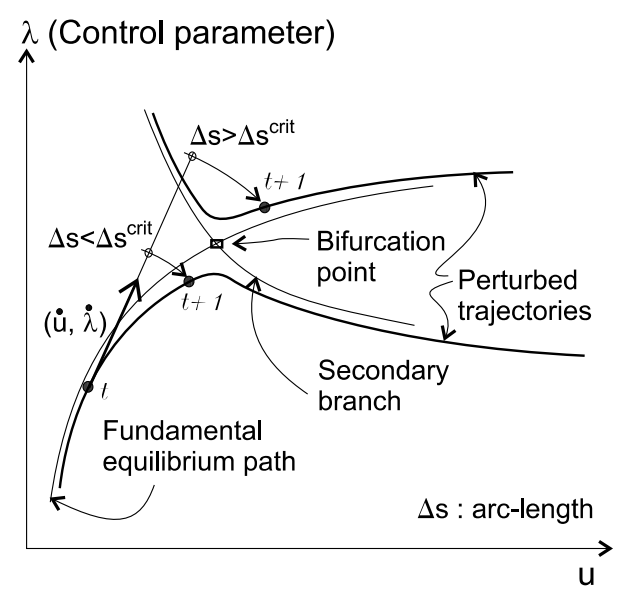

Fig. 5. Bifurcation unfolding.

- The size of the perturbation should be small enough as to keep the physical sense of the numerical response.

- The type of perturbation has to unfold the appropriate branch among the ones emanating from the bifurcation point.

In this section a methodology to unfold bifurcations in continuum material failure, by introducing a viscous perturbation into the failure surfaces, and their beneficial effects on the robustness of the numerical simulation is tackled.

\subsection{Discrete viscous perturbation method}

\subsubsection{Nodal enrichment (variational) formulation}

Let us consider the BVP in Eqs. (32)-(34) now in a total format:

\section{GIVEN:}

$$
\begin{aligned}
& \mathscr{V}_{\eta}:=\left\{\boldsymbol{\eta}=\overline{\boldsymbol{\eta}}+H_{S} \tilde{\boldsymbol{\eta}}^{0} ; \overline{\boldsymbol{\eta}},\left.\tilde{\boldsymbol{\eta}}^{0} \in\left[H^{1}(\Omega)\right]^{n_{\mathrm{dim}}} \tilde{\boldsymbol{\eta}}^{0}\right|_{\Gamma_{u}}=\left.\mathbf{0} \overline{\boldsymbol{\eta}}\right|_{\Gamma_{u}}=\mathbf{u}^{*}\right\}, \\
& \mathscr{V}_{\eta}^{0}:=\left\{\boldsymbol{\eta}=\overline{\boldsymbol{\eta}}^{0}+H_{S} \tilde{\boldsymbol{\eta}}^{0} ; \overline{\boldsymbol{\eta}}^{0},\left.\tilde{\boldsymbol{\eta}}^{0} \in\left[H^{1}(\Omega)\right]^{n_{\operatorname{dim}}} \tilde{\boldsymbol{\eta}}^{0}\right|_{\Gamma_{u}}=\left.\mathbf{0} \overline{\boldsymbol{\eta}}^{0}\right|_{\Gamma_{u}}=\mathbf{0}\right\} .
\end{aligned}
$$

FIND:

$$
\mathbf{u} \in \mathscr{V}_{\eta} ; \quad \mathbf{u}=\overline{\mathbf{u}}+H_{S}[[\mathbf{u}]]
$$

\section{SUCH THAT:}

$$
\int_{\Omega} \nabla^{S} \boldsymbol{\eta}^{0}: \boldsymbol{\sigma} \mathrm{d} \Omega-\left[\int_{\Omega} \boldsymbol{\eta}^{0} \cdot \mathbf{b} \mathrm{d} \Omega+\int_{\Gamma_{\sigma}} \boldsymbol{\eta}^{0} \cdot \mathbf{t}^{*} \mathrm{~d} \Gamma\right]=0 \quad \forall \boldsymbol{\eta}^{0} \in \mathscr{V}_{\eta}^{0},
$$

and the introduction of a perturbation in terms of a set of viscous tractions $\tilde{\mathbf{t}}(\mathbf{x}, t)$ at the failure surface $S$ of the form:

$$
\tilde{\mathbf{t}}(\mathbf{X}, t)=\gamma[[\dot{\mathbf{u}}]](\mathbf{x}, t) \quad \forall \mathbf{X} \in S
$$

where $\gamma>0(\gamma \simeq 0)$ is a perturbing viscosity, which is supposed to be small enough to provide a negligible additional dissipation and to keep the inviscid character of the numerical response. The structure of the 
viscous tractions in Eq. (52) makes them active immediately after the local bifurcation time $t_{\mathrm{B}}(\mathbf{x})$ so that loss of ellipticity is not inhibited. Inserting those tractions into the original variational equation (51d) (Virtual Work Principle) yields

$$
\int_{\Omega} \nabla^{S} \boldsymbol{\eta}^{0}: \boldsymbol{\sigma} \mathrm{d} \Omega-[\int_{\Omega} \boldsymbol{\eta}^{0} \cdot \mathbf{b} \mathrm{d} \Omega+\int_{\Gamma_{\sigma}} \boldsymbol{\eta}^{0} \cdot \mathbf{t}^{*} \mathrm{~d} \Gamma+\int_{S} \boldsymbol{\eta}^{0} \cdot \underbrace{\gamma[[\dot{\mathbf{u}}]]}_{\tilde{\mathbf{t}}} \mathrm{d} S]=0 \forall \boldsymbol{\eta}^{0} \in \mathscr{V}_{\eta}^{0},
$$

and the corresponding Euler-Lagrange equations can be obtained, after standard operations, as:

$$
\begin{aligned}
& \nabla \cdot \boldsymbol{\sigma}+\mathbf{b}=\mathbf{0} \quad \text { in } \Omega \backslash \mathscr{S} \quad \text { (internal equilibrium), } \\
& \boldsymbol{\sigma} \cdot \boldsymbol{v}=\mathbf{t}^{*} \quad \text { on } \Gamma_{\sigma} \quad(\text { external equilibrium }), \\
& \boldsymbol{\sigma}_{\Omega+} \cdot \mathbf{n}-\boldsymbol{\sigma}_{\Omega^{-}} \cdot \mathbf{n}=\gamma[[\dot{\mathbf{u}}]] \quad \text { on } \mathscr{S} \quad\left\{\begin{array}{l}
\text { perturbed } \\
\text { outer traction continuity, }
\end{array}\right. \\
& \boldsymbol{\sigma}_{\Omega^{+}} \cdot \mathbf{n}-\boldsymbol{\sigma}_{S} \cdot \mathbf{n}=\gamma[[\dot{\mathbf{u}}]] \quad \text { on } \mathscr{S} \quad\left\{\begin{array}{l}
\text { perturbed } \\
\text { inner traction continuity, }
\end{array}\right.
\end{aligned}
$$

where it can be observed that the original traction continuity equations (30d) and (30e) are now perturbed in Eqs. (54c) and (54d) by the introduced viscous term $\gamma[[\dot{\mathbf{u}}]]$. As the perturbing viscosity $\gamma$ tends to zero the perturbing force (52) also tends to zero and the original problem is recovered.

However, the introduction of the viscous perturbation has relevant consequences on the uniqueness of the problem. In fact, let us consider two possible solutions of problem (51) whose differences $(\cdot)^{(2)}-(\cdot)^{(1)}$, in terms of the displacements, strains and stresses are:

$$
\begin{aligned}
& \triangle \mathbf{u}(\mathbf{x}, t):=\mathbf{u}^{(2)}(\mathbf{x}, t)-\mathbf{u}^{(1)}(\mathbf{x}, t), \\
& \triangle[[\dot{\mathbf{u}}]](\mathbf{x}, t):=[[\dot{\mathbf{u}}]]^{(2)}(\mathbf{x}, t)-[[\dot{\mathbf{u}}]]^{(1)}(\mathbf{x}, t), \\
& \triangle \boldsymbol{\varepsilon}(\mathbf{x}, t):=\boldsymbol{\varepsilon}^{(2)}(\mathbf{x}, t)-\boldsymbol{\varepsilon}^{(1)}(\mathbf{x}, t), \\
& \triangle \boldsymbol{\sigma}(\mathbf{x}, t):=\boldsymbol{\sigma}^{(2)}(\mathbf{x}, t)-\boldsymbol{\sigma}^{(1)}(\mathbf{x}, t) .
\end{aligned}
$$

Since both are solutions of the variational equation (53) one can write:

$$
\begin{array}{ll}
\int_{\Omega} \nabla^{S} \boldsymbol{\eta}^{0}: \boldsymbol{\sigma}^{(2)} \mathrm{d} \Omega-\left[\int_{\Omega} \boldsymbol{\eta}^{0} \cdot \mathbf{b} \mathrm{d} \Omega+\int_{\Gamma_{\sigma}} \boldsymbol{\eta}^{0} \cdot \mathbf{t}^{*} \mathrm{~d} \Gamma+\int_{S} \boldsymbol{\eta}^{0} \cdot \gamma[[\dot{\mathbf{u}}]]^{(2)} \mathrm{d} S\right]=0 & \forall \boldsymbol{\eta}^{0} \in \mathscr{V}_{\eta}^{0}, \\
\int_{\Omega} \boldsymbol{\nabla}^{S} \boldsymbol{\eta}^{0}: \boldsymbol{\sigma}^{(1)} \mathrm{d} \Omega-\left[\int_{\Omega} \boldsymbol{\eta}^{0} \cdot \mathbf{b} \mathrm{d} \Omega+\int_{\Gamma_{\sigma}} \boldsymbol{\eta}^{0} \cdot \mathbf{t}^{*} \mathrm{~d} \Gamma+\int_{S} \boldsymbol{\eta}^{0} \cdot \gamma[[\dot{\mathbf{u}}]]^{(1)} \mathrm{d} S\right]=0 & \forall \boldsymbol{\eta}^{0} \in \mathscr{V}_{\eta}^{0},
\end{array}
$$

and subtraction of both equations reads

$$
\int_{\Omega} \nabla^{S} \boldsymbol{\eta}^{0}: \triangle \boldsymbol{\sigma} \mathrm{d} \Omega-\int_{S} \boldsymbol{\eta}^{0} \cdot \gamma \Delta[[\dot{\mathbf{u}}]] \mathrm{d} S=0 \quad \forall \boldsymbol{\eta}^{0} \in \mathscr{V}_{\eta^{0}}^{0}
$$

Let us now assume the existence of a bifurcation time, $t_{\mathrm{B}}$, in the time interval of interest, $t_{\mathrm{B}} \in[0, T]$, at which uniqueness is lost and two branches emanate from the fundamental one featuring:

$$
\left.\begin{array}{l}
{[[\dot{\mathbf{u}}]]^{(2)}\left(\mathbf{x}, t_{\mathrm{B}}\right) \neq[[\dot{\mathbf{u}}]]^{(1)}\left(\mathbf{x}, t_{\mathrm{B}}\right)} \\
\triangle[[\dot{\mathbf{u}}]]_{\mathrm{B}}(\mathbf{x}):=[[\dot{\mathbf{u}}]]^{(2)}\left(\mathbf{x}, t_{\mathrm{B}}\right)-[[\dot{\mathbf{u}}]]^{(1)}\left(\mathbf{x}, t_{\mathrm{B}}\right) \neq \mathbf{0}
\end{array}\right\} \text { for some } \mathbf{x} \in S .
$$


Since at $t_{\mathrm{B}}$ the solution is still unique one can write

$$
\triangle \boldsymbol{\sigma}\left(\mathbf{x}, t_{\mathrm{B}}\right)=\Delta \boldsymbol{\varepsilon}\left(\mathbf{x}, t_{\mathrm{B}}\right)=\Delta \mathbf{u}\left(\mathbf{x}, t_{\mathrm{B}}\right)=\mathbf{0} \quad \forall x \in \Omega .
$$

For the specific choice $\boldsymbol{\eta}^{0}=\Delta[[\dot{\mathbf{u}}]]_{\mathrm{B}}(\mathbf{x}) \in \mathscr{V}_{\eta}^{0}$ substitution in Eq. (57) for $t=t_{\mathrm{B}}$ reads:

$$
\begin{gathered}
\int_{\Omega} \nabla^{S}\left(\Delta[[\dot{\mathbf{u}}]]_{\mathrm{B}}\right): \underbrace{\Delta \boldsymbol{\sigma}\left(\mathbf{x}, t_{\mathrm{B}}\right)}_{=0} \mathrm{~d} \Omega-\int_{S} \gamma\left(\Delta[[\dot{\mathbf{u}}]]_{\mathrm{B}}(\mathbf{x}) \cdot \Delta[[\dot{\mathbf{u}}]]_{\mathrm{B}}(\mathbf{x})\right) \mathrm{d} \Gamma \\
=-\int_{S} \gamma\left\|\Delta[[\dot{\mathbf{u}}]]_{\mathrm{B}}(\mathbf{x})\right\|^{2} \mathrm{~d} \Gamma=0 \Rightarrow \Delta[[\dot{\mathbf{u}}]]_{\mathrm{B}}(\mathbf{x})=0 \quad \forall \mathbf{x} \in S
\end{gathered}
$$

which is in contradiction with Eq. (58). Therefore, the introduction of the viscous perturbation (52) precludes any bifurcation in terms of the displacement jump $[[\mathbf{u}]]$ at the failure surface $S$.

\subsubsection{Elemental enrichment formulation}

Let us now consider Eq. (43) (in total format) perturbed with the viscous traction (52):

$$
\int_{S} \tilde{\boldsymbol{\eta}} \cdot\left(\boldsymbol{\sigma}_{\Omega \backslash S}(\mathbf{x}, t)-\boldsymbol{\sigma}_{S}(\mathbf{x}, t)\right) \cdot \mathbf{n} \mathrm{d} S+\int_{S} \tilde{\boldsymbol{\eta}} \cdot \underbrace{\gamma[[\dot{\mathbf{u}}]](\mathbf{x}, t)}_{\hat{\mathbf{t}}} \mathrm{d} S=\mathbf{0} \quad \forall \tilde{\boldsymbol{\eta}} \in L_{2}(S),
$$

in such a way that for $\gamma \rightarrow 0$ the original equation is recovered, and the inner traction continuity equation (30e) is imposed in weak form. Let us consider the bifurcation time $t_{\mathrm{B}}$ as Eqs. (58) and (59) are fulfilled. Substituting solutions $(\cdot)^{(1)}$ and $(\cdot)^{(2)}$ into Eq. (62) and subtracting the results leads to

$$
\int_{S} \tilde{\boldsymbol{\eta}} \cdot(\underbrace{\Delta \boldsymbol{\sigma}_{\Omega \backslash S}\left(\mathbf{x}, t_{\mathrm{B}}\right)}_{=\mathbf{0}}-\underbrace{\left.\Delta \boldsymbol{\sigma}_{S}\left(\mathbf{x}, t_{\mathrm{B}}\right)\right)}_{=\mathbf{0}} \cdot \mathbf{n} \mathrm{d} S+\int_{S} \tilde{\boldsymbol{\eta}} \cdot \gamma \Delta[[\dot{\mathbf{u}}]]\left(\mathbf{x}, t_{\mathrm{B}}\right) \mathrm{d} S=\mathbf{0} \quad \forall \tilde{\boldsymbol{\eta}} \in L_{2}(S),
$$

and for the particular choice $\tilde{\boldsymbol{\eta}}=\Delta[[\dot{\mathbf{u}}]]\left(\mathbf{x}, t_{\mathrm{B}}\right)=\Delta[[\dot{\mathbf{u}}]]_{\mathrm{B}}(\mathbf{x}) \in L_{2}(S)$ in Eq. (63)

$$
\int_{S} \gamma \Delta[[\dot{\mathbf{u}}]]_{\mathrm{B}}(\mathbf{x}) \cdot \Delta[[\dot{\mathbf{u}}]]_{\mathrm{B}}(\mathbf{x}) \mathrm{d} S=\int_{S} \gamma\left\|\Delta[[\dot{\mathbf{u}}]]_{\mathrm{B}}(\mathbf{x})\right\|^{2} \mathrm{~d} S=0 \Rightarrow \Delta[[\dot{\mathbf{u}}]]_{\mathrm{B}}(\mathbf{x})=\mathbf{0} \quad \forall \mathbf{x} \in S
$$

which contradicts Eq. (58). Once again the introduction of the viscous force precludes bifurcations in terms of the displacement jump at the failure surface. This states the beneficial effects of the viscous perturbation method in unfolding such a type of bifurcations.

\subsubsection{Time discretization-critical time step}

In the context of a time (or pseudo-time) advancing algorithm, since progression along the equilibrium path in the action-response space is discrete, it is no longer possible to guarantee, for a given length of the time step $\Delta s(\Delta t)$, the uniqueness of the algorithmic response even for the unfolded (perturbed) problem. Even if the solution at time $t$ lies on the stable branch, if the time step is too large in comparison with the perturbation, there might be more than one solution at time $t+\Delta t$ and the iterative solution might fall in the unstable branch (see Fig. 5). This translates into loss of convergence of the iterative process.

However, it is possible to determine the critical length of the time step to assure algorithmic uniqueness. Let us consider the finite element formulation based on elemental enrichment presented in Section 4.3. The tangent matrix in the linearized system (50a) can be written as

$$
\left[\begin{array}{ll}
\int_{\Omega \backslash S}[\mathbf{B}]^{\mathrm{T}}[\mathscr{D}][\mathbf{B}] \mathrm{d} \Omega & \int_{\Omega \backslash S}[\mathbf{B}]^{\mathrm{T}}[\mathscr{D}][\mathbf{G}] \mathrm{d} \Omega \\
\int_{\Omega}\left[\mathbf{G}^{*}\right]^{\mathrm{T}}[\mathscr{D}][\mathbf{B}] \mathrm{d} \Omega & \int_{\Omega}\left[\mathbf{G}^{*}\right]^{\mathrm{T}}[\mathscr{D}][\mathbf{G}] \mathrm{d} \Omega
\end{array}\right]_{t+\Delta t} \stackrel{\text { def }}{=} \underbrace{\left[\begin{array}{ll}
\mathbf{K}_{d d} & \mathbf{K}_{d \beta} \\
\mathbf{K}_{\beta d} & \mathbf{K}_{\beta \beta}
\end{array}\right]}_{\left[\mathbf{K}_{t+\Delta t}\right]},
$$


where $\left[\mathbf{K}_{t+\Delta t}\right]$ stands for the tangent stiffness matrix at time $t+\Delta t$. In particular submatrix $\mathbf{K}_{\beta \beta}$ associated to the enriching degrees of freedom has the following diagonal-band structure:

$$
\left[\mathbf{K}_{\beta \beta}\right]=\left[\begin{array}{ccccc}
\mathbf{K}_{\beta \beta}^{1} & & & & \\
& \cdot & & \mathbf{0} & \\
& & \mathbf{K}_{\beta \beta}^{e} & & \\
& \mathbf{0} & & \cdot & \\
& & & & \mathbf{K}_{\beta \beta}^{n_{\mathrm{enr}}}
\end{array}\right],
$$

where $\mathbf{K}_{\beta \beta}^{e}$ stands for a $\left(n_{\mathrm{dim}} \times n_{\mathrm{dim}}\right)$ matrix associated to the discontinuous degrees of freedom $\boldsymbol{\beta}^{e}$ of element $e$. The introduction of the viscous perturbation force $\tilde{\mathbf{t}}_{S}=\gamma[[\dot{\mathbf{u}}]]\left(\Rightarrow \dot{\mathbf{t}}_{S}=\gamma[[\ddot{\mathbf{u}}]]\right)$ into the rate version of Eq. (62) reads

$$
\int_{S} \tilde{\boldsymbol{\eta}} \cdot\left[\dot{\boldsymbol{\sigma}}_{\Omega \backslash S}(\mathbf{x}, t+\Delta t)-\dot{\boldsymbol{\sigma}}_{S}(\mathbf{x}, t+\Delta t)\right] \cdot \mathbf{n} \mathrm{d} S+\int_{S} \tilde{\boldsymbol{\eta}} \cdot \gamma \underbrace{\left[\frac{[\dot{\mathbf{u}}]](\mathbf{x}, t+\Delta t)-[[\dot{\mathbf{u}}]](\mathbf{x}, t)}{\Delta t}\right.}_{[[\dot{u}](\mathbf{x}, t+\Delta t)} \mathrm{d} S=0 \quad \forall \tilde{\boldsymbol{\eta}} \in L_{2}(S),
$$

and the subsequent spatial discretization in Eqs. (49) leads, after standard operations, to the following structure of the linearized problem:

$$
\underbrace{\left[\begin{array}{cc}
\mathbf{K}_{d d} & \mathbf{K}_{d \beta} \\
\mathbf{K}_{\beta d} & \mathbf{K}_{\beta \beta}+(\gamma / \Delta t) \mathbf{1}
\end{array}\right]}_{\left[\tilde{\mathbf{K}}_{t+\Delta t}\right]} \underbrace{\left\{\begin{array}{c}
\dot{\mathbf{d}}_{t+\Delta t} \\
\dot{\boldsymbol{\beta}}_{t+\Delta t}
\end{array}\right\}}_{\left\{\dot{\boldsymbol{a}}_{t+\Delta t}\right\}}=\underbrace{\left\{\begin{array}{c}
\dot{\mathbf{f}}_{\mathbf{d}} \\
(\bar{\gamma} / \Delta t) \dot{\boldsymbol{\beta}}_{t}
\end{array}\right\}}_{\left\{\dot{\mathbf{F}}_{t+\Delta t}\right\}}
$$

where $\left[\tilde{\mathbf{K}}_{t+\Delta t}\right]$ and $\left\{\dot{\tilde{\mathbf{F}}}_{t+\Delta t}\right\}$ stand, respectively, for the tangent stiffness matrix and incremental forces vector for the unfolded problem. A non-unique solution at time $t+\Delta t$ is characterized by the existence of several solutions of Eq. (68) for the same value of $\dot{\tilde{\mathbf{F}}}_{t+\Delta t}$. Therefore, the difference of both solutions $\Delta \dot{\mathbf{a}}_{t+\Delta t}:=\dot{\mathbf{a}}_{t+\Delta t}^{(2)}-\dot{\mathbf{a}}_{t+\Delta t}^{(1)} \neq \mathbf{0}$ fulfills:

$$
\left[\tilde{\mathbf{K}}_{t+\Delta t}\right]\left\{\Delta \dot{\mathbf{a}}_{t+\Delta t}\right\}=\{\mathbf{0}\}
$$

which states the singularity of $\left[\tilde{\mathbf{K}}_{t+\Delta t}\right]$. Therefore, the value of $\Delta t$ that guarantees positive definiteness of $\left[\tilde{\mathbf{K}}_{t+\Delta t}\right]$ precludes any bifurcation. Since submatrix $\left[\mathbf{K}_{d d}\right]$ in Eq. (68) can be shown to be positive, an approximate value of $\Delta t=\Delta t^{\text {crit }}$ can be obtained by imposing positive definiteness of the symmetric part of all the diagonal submatrices $\left[\tilde{\mathbf{K}}_{\beta \beta}\right]^{e}$ in Eq. (68) associated to the enriching degrees of freedom, i.e.,

$$
\operatorname{sym}\left(\left[\tilde{\mathbf{K}}_{\beta \beta}\right]^{e}\right)=\operatorname{sym}\left(\left[\mathbf{K}_{\beta \beta}^{e}+(\gamma / \Delta t) \mathbf{1}_{n_{\operatorname{dim}} \times n \operatorname{dim}}\right]\right) \rightarrow \text { positive definite } \forall e \in\left\{1, \ldots, n_{\mathrm{enr}}\right\}
$$

or, equivalently,

$$
\lambda_{\min }^{e}+\frac{\gamma}{\Delta t}>0 \quad \forall e \in\left\{1, \ldots, n_{\mathrm{enr}}\right\}
$$

where $\lambda_{\min }^{e}$ stands for the minimum eigenvalue of $\operatorname{sym}\left(\left[\mathbf{K}_{\beta \beta}^{e}\right]\right)$. From Eq. (71) the following value for $\Delta t^{\text {crit }}$ emerges:

$$
\Delta t^{\mathrm{crit}}=\min _{e} \frac{\gamma}{\left\langle-\lambda_{\min }^{e}\right\rangle} \quad \forall e \in\left\{1, \ldots, n_{\mathrm{enr}}\right\},
$$

where $\langle\cdot\rangle$ stands for the ramp function. Therefore, a time step $\Delta t<\Delta t^{\text {crit }}$ guarantees uniqueness of the solution at time $t+\Delta t$ which shall lie on the right equilibrium path. Nearby bifurcation points, $\Delta t^{\text {crit }}$ tends to be small and proportional to the perturbation viscosity as pointed out in Eq. (72). At regular points of the equilibrium path, $\Delta t^{\text {crit }} \rightarrow \infty$ and the length of the time step is determined by reasons other than uniqueness. 


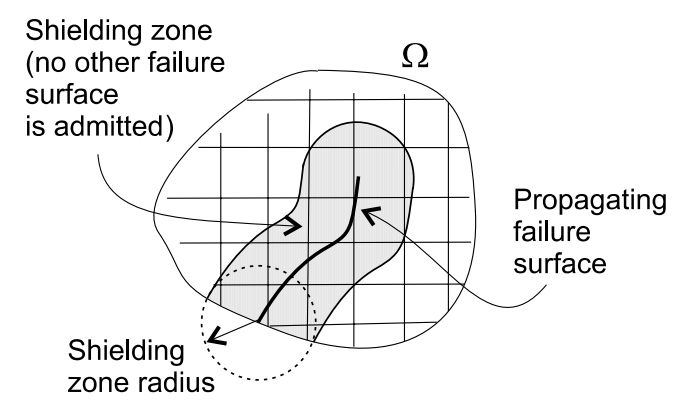

Fig. 6. Shielding zone around a propagating failure surface.

Remark 7. In the context of the considered quasi-static problems, time $t$ is just a parameter controlling the advance along the equilibrium path that, for practical purposes, can be substituted in the previous derivation by the actual control parameter, i.e., the load parameter $\lambda^{*}$, in an incremental load advancing method, or the arc-length parameter $s$, in arc-length type continuation methods. Therefore, for practical implementation purposes $\Delta t$ and $\Delta t^{\text {crit }}$ in Eqs. (68)-(72) must be substituted by $\Delta \lambda^{*}, \Delta \lambda^{* \text { crit }}$ or $\Delta s, \Delta s^{\text {crit }}$ depending on the load control method (see Fig. 5).

\subsubsection{Shielding zone}

The finite element methodology presented above is not thought for the case of several failure surfaces crossing each other inside the same element. However, in the numerical experiments performed by the authors it has been, sometimes, observed during nonlinear iterations, a secondary spurious failure surface attempting to cross one of the primary failure surfaces. This situation can result in a sort of numerical locking and a subsequent loss of convergence. To circumvent this problem, in the numerical examples presented below, the elements inside a shielding zone (whose width is defined by the user) around an existing propagating failure surface can not be crossed by any external surface (see Fig. 6). This prevents the formation of a number of spurious secondary failure surfaces, around the primary ones, that give rise to numerical difficulties but not providing any gain in the physical insight of the problem.

\section{Representative numerical simulations}

In the following sections several examples illustrate the behavior of the preceding methodologies in material failure simulation. For this purpose the following ingredients have been considered:

- An isotropic continuum damage model (see [21]) for a detailed description.

- The quadrilateral 2D finite element with an embedded discontinuity based on elemental enrichment described in Section 4.3.

- The discrete viscous perturbation method described in Section 5.1 when necessary. The control of the time step length based on Eq. (72) is considered as well.

\subsection{Smoothing a singular sharp point}

In Fig. 7 the following numerical experiment is sketched: a bar, discretized in 10 elements, is stretched by applying a uniform set of forces at its right-hand side (see Fig. 7a). The properties of the material are shown in the same figure, where $E$ is Young's modulus, $v$ Poisson's ratio, $G_{\mathrm{f}}$ the fracture energy and $\sigma_{\mathrm{u}}$ the peak stress. 


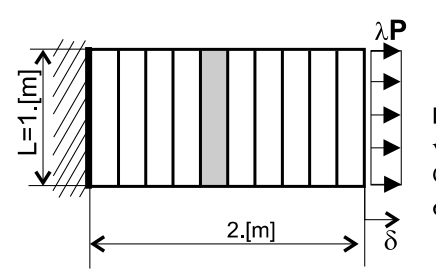

(a)

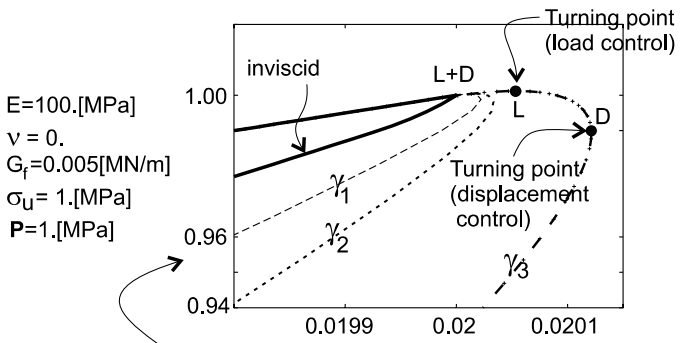

(c)

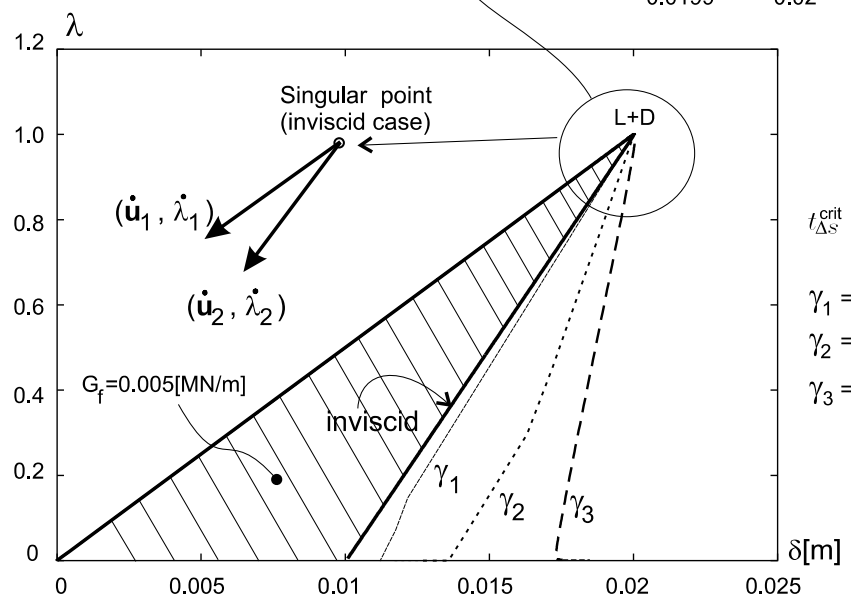

$t_{\Delta s}^{\text {crit }}=0.45 E-5[p s e g]$

$\gamma_{1}=0.65^{*}\left(E E_{\Delta S}^{\text {crit } / L)}\right.$

$\gamma_{2}=1.30^{*}\left(E_{\Delta s}^{\text {crit } / L)}\right.$

$\gamma_{3}=13.0^{*}\left(E t_{\Delta S}^{\text {crit }} / L\right)$

(b)

Fig. 7. Smoothing of a sharp singular point by a viscous perturbation: (a) geometric and material data; (b) load parameter vs. displacement curves; (c) close up view of the peak of the curves.

At the central element, the peak stress is slightly reduced in order to trigger the material failure in that element. In consequence, the force-displacement curve exhibits an initial elastic branch, until the peak stress is reached at the central element, and a subsequent softening branch (see Fig. 7b). The area under the force-displacement curve is proportional to the fracture energy. Therefore, for sufficiently small values of $G_{\mathrm{f}}$ the descending branch has a positive slope. Under these conditions the upper point in the equilibrium path becomes, simultaneously, a turning point under load control $\mathbf{L}$ (limit point) and a turning point under displacement control $\mathbf{D}$ (snap-back point). As a result, the solution at this point in terms of the state variables $\mathbf{x}^{*} \equiv[\dot{\mathbf{u}}, \dot{\lambda}]^{\mathrm{T}}$ is not unique, since there are two different solutions, $\mathbf{x}_{1}^{*} \equiv\left[\dot{\mathbf{u}}_{1}, \dot{\lambda}_{1}\right]^{\mathrm{T}}$ and $\mathbf{x}_{2}^{*} \equiv\left[\dot{\mathbf{u}}_{2}, \dot{\lambda}_{2}\right]^{\mathrm{T}}$ (see Fig. 7b). In consequence, displacement control, force control and standard arc-length continuation methods fail to pass this sharp singular point.

Remark 8. Notice that this type of instability has a structural nature (it comes from the small value of $G_{\mathrm{f}}$ with respect to the strain energy stored in the bar) and can not be attributed to the type of enrichment (nodal or elemental) used to capture the discontinuity.

The inclusion of the viscous perturbation dramatically changes this situation. In Fig. $7 \mathrm{~b}$ the forcedisplacement curve, for decreasing values of $\gamma$, is presented together with the theoretical inviscid (unperturbed) result. There it can be checked:

(1) The effect of decreasing perturbations $(\gamma \rightarrow 0)$ making the results, as expected, converge to an inviscid (theoretical) limit. 
(2) The benefits of the viscous perturbation on unfolding and smoothing the sharp point. This is clearly stated in Fig. 7c showing a close up view of the peak of the curves. Increasing values of the viscosity $\gamma$ move both turning points, $\mathbf{L}$ and $\mathbf{D}$, away from each resulting in a unique solution $\mathbf{x}^{*} \equiv[\dot{\mathbf{u}}, \dot{\lambda}]^{\mathrm{T}}$ all along the equilibrium path. As a result the unfolded equilibrium path can be now reproduced with no difficulties using standard arc-length procedures.

Although $\Delta t^{\text {crit }} \rightarrow 0$ as $\gamma \rightarrow 0$, for any $\gamma>0$ there is a corresponding critical time step that assures algorithmic uniqueness according to Eq. (72). The smaller are the values of $\gamma$ the larger will be the required number of time steps to cross the singular point, but algorithmic uniqueness is always assured for $\gamma>0$ while keeping convergence to the inviscid solution.

\subsection{Unfolding a bifurcation point}

The numerical example simulates the propagation of two cracks within a bulk of concrete-like material, constrained by two infinitely rigid plates forced to open as indicated in Fig. 8a in a plane strain setting. The considered material properties for the concrete are shown in the same figure and the finite element mesh is displayed in Fig. 8b. Observe that the geometry, finite element mesh and loading system are perfectly symmetric with respect to the central vertical axis of the specimen. In order to induce a perfectly symmetric couple of cracks, two elements (labelled 1 and $\mathbf{2}$ in Fig. 8b) have a small (the same for both) perturbation on the peak stress $\sigma_{y}$. Therefore, from the symmetry of the problem one should expect a fundamental equilibrium path characterized by two symmetric parallel cracks developing from points $\mathbf{1}$ and $\mathbf{2}$. This is the solution, presented in Fig. 8c, which is numerically obtained when no viscous perturbation is included. At point $\mathbf{A}$, the peak stress is simultaneously attained at elements $\mathbf{1}$ and $\mathbf{2}$ of the specimen. In addition, at the neighborhood of point $\mathbf{B}$, negative pivots in the stiffness matrix indicate the presence of a singular point. This corresponds to a bifurcation where a secondary branch, characterized by only one propagating crack, the other arresting, emanates. Indeed, from the structural failure point of view, this is the most interesting failure mode since, for a real specimen, any physical perturbation breaking the theoretical symmetry would result in that only-one-crack solution. Besides, since the total crack-length in this case is smaller, one should expect about half the dissipation (area under the force-displacement curve) for this solution in comparison with the previous one and, consequently, a lower peak force characterizing that one-crack-mode as the most unsafe (critical) failure mode.

However, the round off errors are not enough to unfold that bifurcation at point $\mathbf{B}$ and the (inviscid) unperturbed simulation proceeds along the fundamental branch. On the contrary, the introduction of the viscous perturbation always results in the one-crack failure mode. This failure mode is characterized by (see Fig. 8c):

(a) Onset of both cracks at point $\mathbf{A}$ of the force-displacement curve.

(b) Subsequent development of both cracks following the fundamental branch, up to point $\mathbf{B}$ characterizing the peak of the force-displacement curve.

(c) Bifurcation characterized by arrest (unloading) of crack $\mathbf{1}$ and propagation of crack $\mathbf{2}$. The one-crack failure mode is triggered at point $\mathbf{B}$ and holds for the rest of the equilibrium path (point $\mathbf{C}$ in the curve).

This pattern holds independently of the value of the viscous perturbation $\gamma$. As it can be checked in Fig. $8 \mathrm{~d}$, the larger is $\gamma$ the more delayed is the bifurcation (peak point of the load-displacement curve) and the larger is the spurious dissipation due to the viscous mechanism. However, in all cases the triggered failure mode is the one-crack mode displayed in Fig. 8c, and convergence as $\gamma \rightarrow 0$, to the one-crack inviscid solution forced by introduction of an unsymmetrical perturbation in the peak stress only in element 2, can be observed in Fig. 8d. This seems to state that the discrete perturbation method, described 

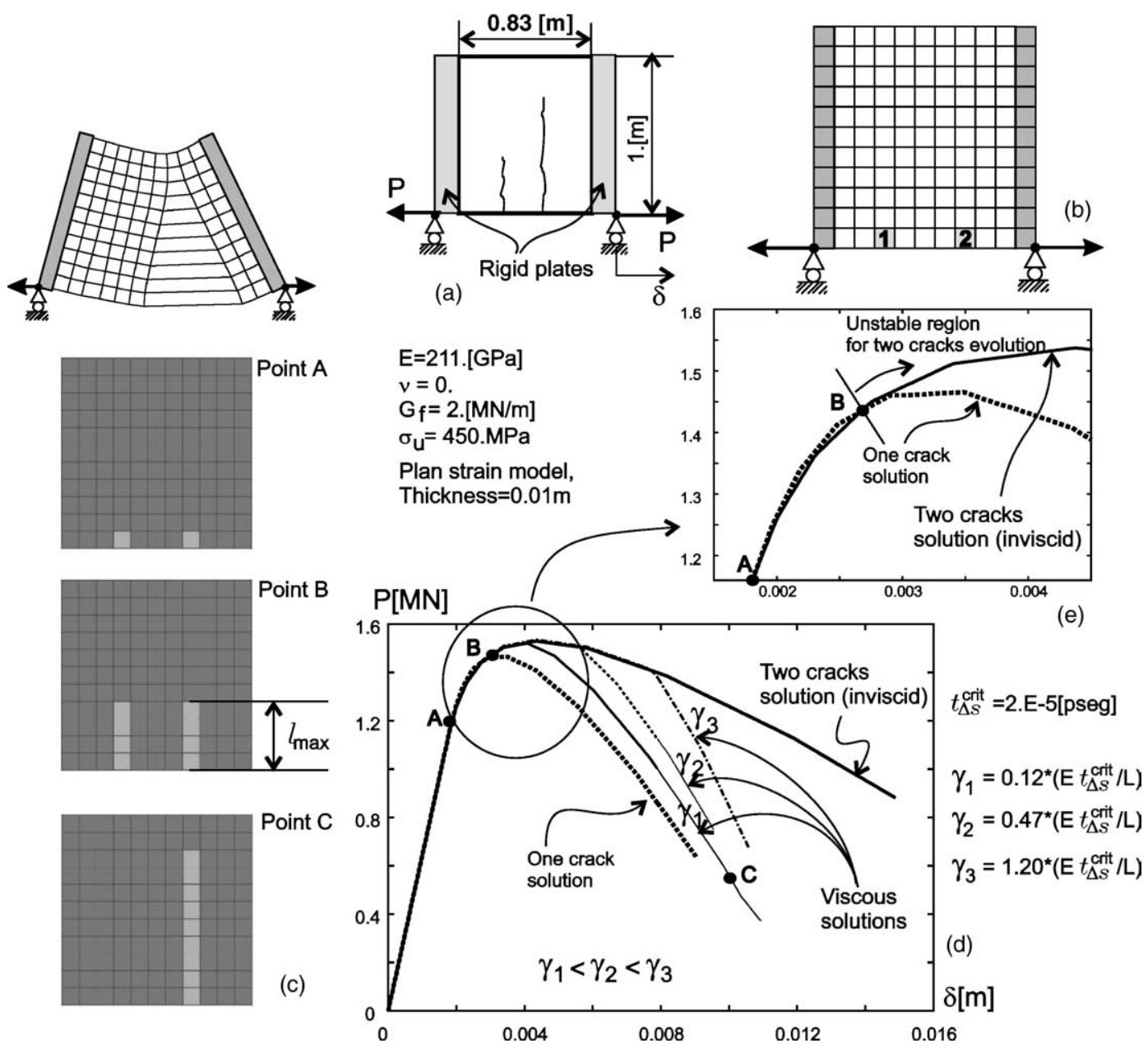

Fig. 8. Unfolding a bifurcation point: (a) geometric and material data; (b) finite element mesh; (c) evolution of the cracks along the equilibrium curve; (d) load vs. displacement curves; (e) close up view.

above, unfolds the bifurcation towards the less dissipative branch thus display the most critical and stable failure mode.

As for which of the two (right- or left-hand-side) cracks gets arrested, for this perfectly symmetric case this seems to be determined by round off errors. In the presented simulations the left-hand-side crack 1 always arrests. However, a small additional perturbation on the peak stress at point $\mathbf{1}$ is enough to revert the crack pattern and make crack 2 arresting.

Once again the use of the critical time step length $\Delta t^{\text {crit }}$ in Eq. (72) assures robustness and uniqueness of the algorithmic solution in the neighborhood of the bifurcation (peak) point.

\subsection{Steel slab debonding from a concrete specimen}

A tension pull test applied to a reinforced concrete specimen is analyzed in this section. This test was inspired by a similar one presented in [31] where the axisymmetric case was considered, while in the numerical simulation presented here the plane strain case is assumed. Besides, and due to the symmetry, 
only one fourth of the specimen is modelled. The dimensions and loads applied to the specimen are displayed in Fig. 9a. An only-tension-damage model [26] was used for the concrete matrix, considering the following material properties: $E_{\mathrm{c}}=2.5 \times 10^{4} \mathrm{MPa}, \sigma_{\mathrm{u}}=3 \mathrm{MPa}, v_{\mathrm{c}}=0.18$, and $G_{\mathrm{f}}=25 \mathrm{~N} / \mathrm{m}$. The reinforcement slab was modelled as an elastic material with $E_{\mathrm{s}}=2.14 \times 10^{5} \mathrm{MPa}, v_{\mathrm{s}}=0.3$.

The bond-slip mechanism that rules the transmission of the tangential stresses to the concrete is modeled by a thin layer of an ideally plastic (J2 plasticity) material at the steel-concrete interface (see Fig. 9b). This bonding material has the following properties: $E_{\mathrm{b}}=2.14 \times 10^{5} \mathrm{MPa}, \sigma_{y \mathrm{~b}}=3 \mathrm{MPa}, v_{\mathrm{b}}=0.3$.

In order to check mesh sensitivity of the results two different finite element meshes A (coarse) and B (fine) have been used for the numerical simulations.

Fig. 9d shows the evolution of the load $P$, applied at the end-face of the steel slab, vs. the displacement at the same point. It exhibits a sharp snap-back characterizing a sudden change in the failure mode. In Fig. 9e a closed up view shows in detail this behavior for both meshes.
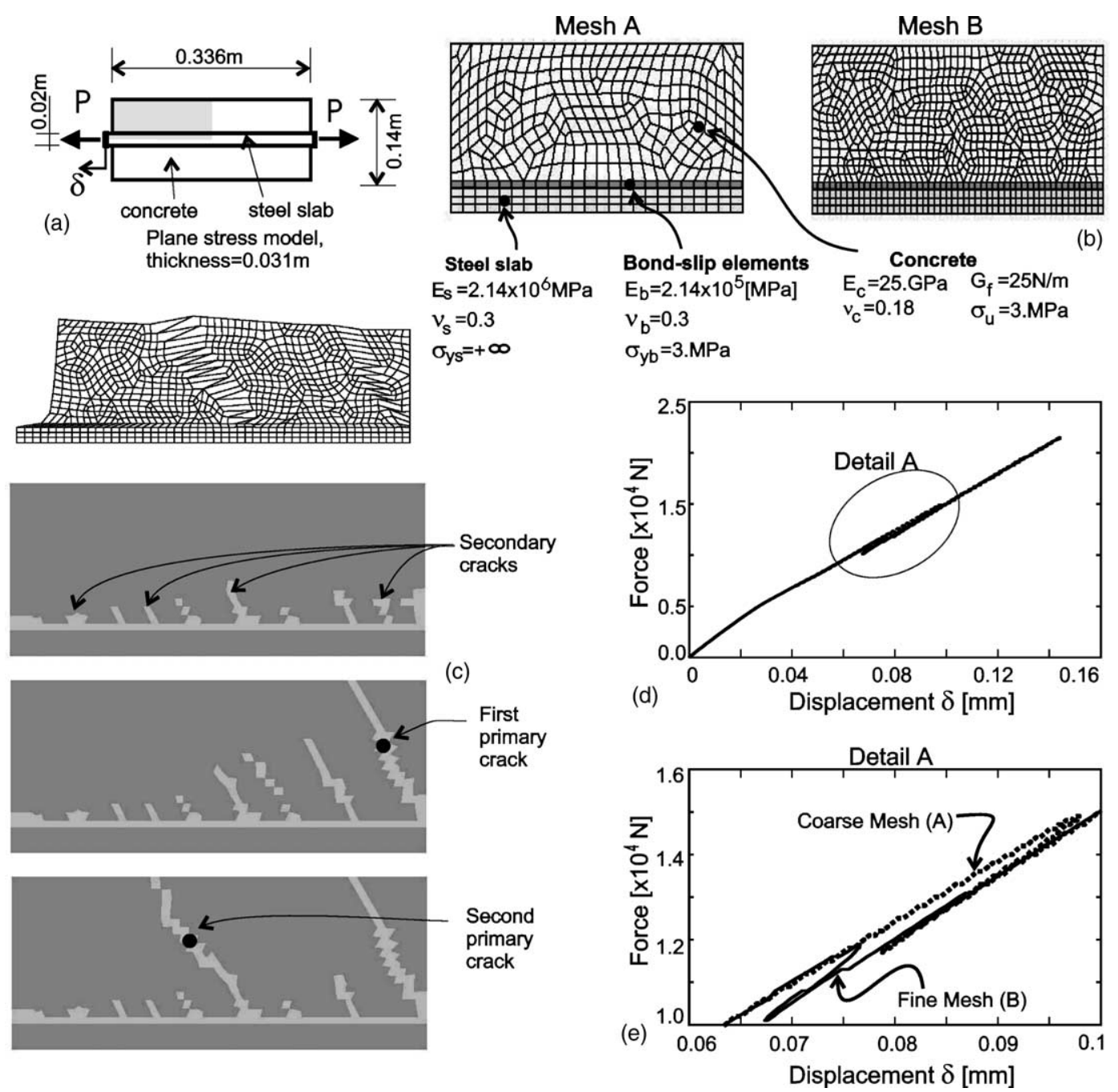

Fig. 9. Steel slab debonding from a concrete specimen: (a) and (b) finite element meshes; (c) evolution of the cracks along the equilibrium curve; (d) load vs. displacement curves; (e) close up view of the snap-back zone. 
The crack pattern exhibits a large number of failure lines. Some of them, that will be termed secondary cracks, are active during a short period of the deformation process an do not consume a relevant amount of energy. Some others, on the contrary, remain active for a large part of the process and dissipate an important part of the supplied energy. They will be termed primary cracks and their capture is the main goal of the simulation since they are supposed to determine the failure mode.

Secondary transverse cracks can be observed in an initial diffuse failure stage, before the snap-back of the equilibrium path takes place (see Fig. 9c). They initially nucleate at the end-face of the specimen but, as the loading increases, they spread out through the total steel-concrete interface length. An initial primary crack onsets and propagates across the concrete section, up to the complete softening of the material in that region (see Fig. 9c). It is responsible for the subsequent loss of loading capacity and triggers the snap-back displayed in the equilibrium path in Fig. 9d.

At this stage, the bulk of concrete is still able to hold an increasing external loading that is transmitted through the steel slab to other sections of the concrete bulk. In fact, a subsequent loading after the snapback induces the propagation of a second primary crack (see Fig. 9c) across the concrete and a severe change in the failure mode since the first primary crack tends to unload. Once the second crack is fully developed the concrete has no remaining strength, the steel plate is almost completely debonded and the specimen would eventually fail by plastic yielding of the steel.

Results, in terms of failure modes and force-displacement curves (see Fig. 9d), obtained with both (fine and coarse) meshes compare reasonably well. For the numerical simulations of this problem, the use of the viscous perturbation method of Section 5.1 became crucial to provide smoothness to the obtained equilibrium path and to prevent loss of uniqueness, this resulting in a robust simulation process.

\section{Concluding remarks}

Throughout this work some recent developments on numerical simulation of continuum material failure have been presented. They aim at increasing the reliability and robustness of numerical modelling of material failure in strong discontinuity settings in the following aspects:

- Characterization of the onset and propagation of local failure. There is a clear connection of loss of material strong ellipticity with the onset of a weak discontinuity as a precursor of a strong discontinuity [22]. In this sense, singularity of the localization tensor and discontinuous bifurcation tools provide physically grounded material failure indicators and directions of propagation of failure, which seem specially suited for strong discontinuity settings. Closed form solutions (exact and approximated) for those indicators, for a wide class of constitutive models used in geomaterials, and not restricted to particular (bi-dimensional plane-stress, plane-strain or three-dimensional) cases have been provided. They have been successfully checked by the authors for several isotropic associative elasto-plastic and damage models where those solutions are exact. Assessment of the approximated results provided by these formula for more general constitutive models is a remaining task.

- Finite element modelling. Two alternatives, nodal and element enrichment, for finite elements with embedded discontinuities presently available for capturing displacement discontinuities have been presented, and their formulations in a continuum strong discontinuity approach (CSDA) have been developed. Which is the most suitable, for the purposes of simulation of material failure, is still a controversial issue out of the scope of this work. The numerical simulations presented in this work in 2D problems have been carried out using elemental enriched elements in a specific finite element code [8]. Additional issues, like crack branching modelling, which would require some, not conceptual but not trivial, modification of those finite element formulations, and applications to 3D problems will be addressed in subsequent works. 
- Instability treatment. Strain softening in continuum models is a source of severe instabilities and ill-posedness of the boundary value problem. The CSDA and the entailed softening regularization procedure contributes to the well-posedness of the problem and to get rid of most of the local instabilities [23]. However, structural uniqueness problems still remain due to the structural softening present in the problem and the failure surfaces interaction. A strategy based on a discrete viscous perturbation at the failure surfaces, of trivial implementation, has been presented, and its performance in several examples has been displayed. Two main benefits might be extracted from this methodology: (a) assure algorithmic uniqueness in terms of the displacement jump, which entails a dramatic increase in the robustness of the numerical simulations keeping the approximation of the results; and (b) the discrete perturbation seems to activate the least energy consuming among the possible combinations in terms of development/arrest of multiple failure surfaces, this providing the most unfavorable solutions in terms of material failure. This fact has not been theoretically proved, but the experiences of the authors in a large number of numerical simulations seem to corroborate it.

\section{Acknowledgements}

Financial support from the Spanish Ministry of Science and Technology, through grant MAT2001-3863C03-03, and from the Catalan Government Research Department, through CIRIT grant 2001-SGR 00262, is gratefully acknowledged.

\section{Appendix A}

Let us consider the following tensors:

$$
\begin{aligned}
& \mathbf{P}=\sum_{i=1}^{i=3} p_{i} \hat{\mathbf{e}}_{i} \otimes \hat{\mathbf{e}}_{i}, \\
& \mathbf{R}=\sum_{i=1}^{i=3} r_{i} \hat{\mathbf{e}}_{i} \otimes \hat{\mathbf{e}}_{i}, \\
& \mathbf{S}=\sum_{i=1}^{i=3} \operatorname{sign}\left(p_{i}\right) \sqrt{p_{i} r_{i}} \hat{\mathbf{e}}_{i} \otimes \hat{\mathbf{e}}_{i}
\end{aligned}
$$

such that $p_{i} r_{i} \geqslant 0$ and, therefore, $\operatorname{sign}\left(p_{i}\right)=\operatorname{sign}\left(r_{i}\right), i \in\{1,2,3\}$. Then, for a vector

$$
\begin{aligned}
\mathbf{n}=\sum_{i=3}^{i=3} n_{i} \hat{\mathbf{e}}_{i}: & \\
(\mathbf{n} \cdot \mathbf{P} \cdot \mathbf{n})(\mathbf{n} \cdot \mathbf{R} \cdot \mathbf{n})-(\mathbf{n} \cdot \mathbf{S} \cdot \mathbf{n})^{2} & =\left(\sum_{i=1}^{i=3} n_{i}^{2} p_{i}\right)\left(\sum_{i=1}^{i=3} n_{i}^{2} r_{i}\right)-\left(\sum_{i=1}^{i=3}\left(n_{i}^{2} \operatorname{sign}\left(p_{i}\right) \sqrt{p_{i} r_{i}}\right)^{2}\right) \\
& =\sum_{i=1}^{i=3}\left(n_{i}^{4} p_{i} r_{i}\right)+\sum_{\substack{k=1 \\
i<j, i \neq k, j \neq k}}^{n_{i}^{2} n_{j}^{2}} \underbrace{\left(p_{i} r_{j}+p_{j} r_{i}\right)}_{\substack{k=3 \\
\text { sign }\left(p_{i}\right)\left|p_{i}\right| \operatorname{sign}\left(r_{j}\right)\left|r_{j}\right| \\
+\operatorname{sign}\left(p_{j}\right)\left|p_{j}\right| \operatorname{sign}\left(r_{i}\right)\left|r_{i}\right|}}
\end{aligned}
$$




$$
\begin{aligned}
& -\sum_{i=1}^{i=3} n_{i}^{4} \underbrace{\operatorname{sign}\left(p_{i}\right)^{2}}_{=1} p_{i} r_{i}-\sum_{\substack{k=1 \\
i<j, i \neq k, j \neq k}}^{k=3} 2 n_{i}^{2} n_{j}^{2} \operatorname{sign}\left(p_{i}\right) \operatorname{sign}\left(p_{j}\right) \sqrt{p_{i} r_{i}} \sqrt{p_{j} r_{j}} \\
& =\sum_{\substack{k=1 \\
i<j, i \neq k, j \neq k}}^{k=3} n_{i}^{2} n_{j}^{2} \operatorname{sign}\left(p_{i}\right) \operatorname{sign}\left(p_{j}\right)\left(\left|p_{i}\right|\left|r_{j}\right|+\left|p_{j}\right|\left|r_{i}\right|\right) \\
& -\sum_{k=1}^{k=3} n_{i}^{2} n_{j}^{2} \operatorname{sign}\left(p_{i}\right) \operatorname{sign}\left(p_{j}\right) \sqrt{p_{i} r_{i}} \sqrt{p_{j} r_{j}} \\
& i<j, i \neq k, j \neq k \\
& =\sum_{\substack{k=1 \\
i<j, i \neq k, j \neq k}}^{k=3} 2 n_{i}^{2} n_{j}^{2} \operatorname{sign}\left(p_{i}\right) \operatorname{sign}\left(p_{j}\right)[\underbrace{\frac{1}{2}\left(\left|p_{i} r_{j}\right|+\left|p_{j} r_{i}\right|\right)}_{\text {arithmetic average }}-\underbrace{\sqrt{\left|p_{i} r_{j}\right|\left|p_{j} r_{i}\right|}}_{\text {geometric average }}] \\
& =\sum_{\substack{k=1 \\
i<j, i \neq k, j \neq k}}^{k=3} 2 n_{i}^{2} n_{j}^{2} \operatorname{sign}\left(p_{i}\right) \operatorname{sign}\left(p_{j}\right) \\
& \times\left[\text { average }_{\text {arith }}-\text { average }_{\text {geom }}\right] \text { average }_{\text {arith }}\left(\left|p_{i} r_{j}\right|,\left|p_{j} r_{i}\right|\right) \\
& =\frac{1}{2}\left(\left|p_{i} r_{j}\right|+\left|p_{j} r_{i}\right|\right) \text { average }_{\text {geom }}\left(\left|p_{i} r_{j}\right|,\left|p_{j} r_{i}\right|\right)=\sqrt[2]{\left(\left|p_{i} r_{j}\right|\left|p_{j} r_{i}\right|\right)} .
\end{aligned}
$$

If $\mathbf{R}=\mathbf{P}$, then

$$
\begin{aligned}
& r_{i}=p_{i} ; \quad r_{j}=p_{j}, \\
& \text { average }_{\text {arith }}=\left|p_{i} p_{j}\right|=\text { average }_{\mathrm{geom}}=\left|p_{i} p_{j}\right| \Rightarrow(\mathbf{n} \cdot \mathbf{P} \cdot \mathbf{n})(\mathbf{n} \cdot \mathbf{R} \cdot \mathbf{n})-(\mathbf{n} \cdot \mathbf{S} \cdot \mathbf{n})^{2}=0 .
\end{aligned}
$$

\section{Appendix B}

Equations for the ellipse and outer Mohr's circle in Fig. 2b are:

$$
\begin{aligned}
& \text { Localization ellipse } \rightarrow \frac{\sigma^{2}}{\frac{Z}{b-a}}+\frac{\tau^{2}}{\frac{Z}{b}}=1, \\
& \text { Outer Mohr's circle } \rightarrow\left\{\begin{array}{l}
(\sigma-c)^{2}+\tau^{2}=R^{2}, \\
c=\frac{s_{1}+s_{3}}{2}, R=\frac{s_{1}-s_{3}}{2} .
\end{array}\right.
\end{aligned}
$$

Since we are looking for the tangent ellipse, there will only be two solutions for the intersection of both locus in terms of points in Mohr's space $\left(\sigma^{*}, \tau^{*}\right),\left(\sigma^{*},-\tau^{*}\right)$ in Fig. 2b. Solving for $\sigma$ in the previous system leads to the quadratic equation:

$$
\underbrace{-\frac{a}{b}}_{p} \sigma^{2}+\underbrace{2 c}_{q} \sigma+\underbrace{\left(R^{2}+\frac{Z}{b}-c^{2}\right)}_{r}=0 .
$$


A unique solution for $\sigma\left(\sigma=\sigma^{*}\right)$ in this equation requires:

$$
\begin{aligned}
& \Delta=q^{2}-4 p r=0 \Rightarrow \mathrm{Z}^{\mathrm{crit}}=b\left[\left(\frac{s_{1}-s_{3}}{2}\right)^{2}+\left(\frac{b}{a}-1\right)\left(\frac{s_{1}+s_{3}}{2}\right)^{2}\right], \\
& \sigma^{*}=-\frac{q}{2 p}=\frac{b}{a} c \Rightarrow \mathbf{n}^{\mathrm{crit}} \cdot \mathbf{S} \cdot \mathbf{n}^{\mathrm{crit}}=\frac{b}{a} c=\frac{b}{a}\left(\frac{s_{1}+s_{3}}{2}\right),
\end{aligned}
$$

where Eq. (24a) has been considered. Since solutions $\left(\sigma^{*}, \tau^{*}\right),\left(\sigma^{*},-\tau^{*}\right)$ lie on the outer Mohr's circle, see Fig. $2 b, \mathbf{n}^{\text {crit }}$ can be characterized (after some straightforward operations) in terms of the eigenvalues $\left(s_{1} \geqslant s_{2} \geqslant s_{3}\right)$ of the symmetric tensor $\mathbf{S}=\operatorname{sign}(\mathbf{P}) \sqrt{\mathbf{P} \cdot \mathbf{R}}$ and the corresponding eigenvectors $\hat{\mathbf{s}}_{1}, \hat{\mathbf{s}}_{2}, \hat{\mathbf{s}}_{3}$ :

$$
\begin{aligned}
& \mathbf{n}^{\text {crit }}=\cos \theta^{\text {crit }} \hat{\mathbf{s}}_{1}+\sin \theta^{\text {crit }} \hat{\mathbf{s}}_{3}, \\
& \tan ^{2} \theta^{\text {crit }}=-\frac{(1-2 a / b) s_{1}+s_{3}}{(1-2 a / b) s_{3}+s_{1}} .
\end{aligned}
$$

\section{References}

[1] J. Alfaiate, L.J. Sluys, Analysis of a compression test on concrete using strong embedded discontinuities, in: F.G. Eberhardsteiner, J. Mang, H.A. Rammerstorfer (Eds.), Proceedings of the Fifth World Congress on Computational Mechanics (WCCM V), Vienna University of Technology, Austria, ISBN 3-9501554-0-6, July 7-12, 2002. Available from <http://wccm.tuwien.ac.at>.

[2] F. Armero, K. Garikipati, Recent advances in the analysis and numerical simulation of strain localization in inelastic solids, in: D.R.J. Owen, E. Onate, E. Hinton (Eds.), Computational Plasticity. Fundamentals and Applications, pp. 547-561, 1995.

[3] F. Armero, K. Garikipati, An analysis of strong discontinuities in multiplicative finite strain plasticity and their relation with the numerical simulation of strain localization in solids, Internat. J. Solids and Structures 33 (20-22) (1996) $2863-2885$.

[4] Z.P. Bazant, Mechanics of distributed cracking, Appl. Mech. Rev. 39 (5) (1986) 675-701.

[5] Z.P. Bazant, L. Cedolin, Stability of Structures, Oxford University Press, 1991.

[6] Z.P. Bazant, J. Planas, Fracture and size effect in concrete and other quasibrittle materials, CRC Press, 1998.

[7] R.I. Borja, A finite element model for strain localization analysis of strongly discontinuous fields based on standard Galerkin approximation, Comput. Methods Appl. Mech. Engrg. (190) (2000) 1529-1549.

[8] M. Cervera, C. Agelet de Saracibar, M. Chiumenti, COMET: a multipurpose finite element code for numerical analysis in solid mechanics, Technical Report, Technical University of Catalonia (UPC), 2001.

[9] M.A. Crisfield, Non-Linear Finite Element Analysis of Solids and Structures, vol. I, John Wiley, England, 1991.

[10] E.N. Dvorkin, A.M. Cuitino, G. Gioia, Finite elements with displacement embedded localization lines insensitive to mesh size and distortions, Internat. J. Numer. Methods Engrg. 30 (1990) 541-564.

[11] A. Hillerborg, Numerical methods to simulate softening and fracture of concrete, in: G.C. Sih, A. Di Tomaso (Eds.), Fracture Mechanics of Concrete: Structural Application and Numerical Calculation, 1985, pp. 141-170.

[12] M. Jirasek, Comparative study on finite elements with embedded discontinuities, Comput. Methods Appl. Mech. Engrg. 188 (2000) 307-330.

[13] M. Jirasek, T. Belytschko, Computational resolution of strong discontinuities, in: H.A. Mang, F.G. Rammerstorfer, J. Eberhardsteiner (Eds.), Proceedings of the Fifth World Congress on Computational Mechanics (WCCM V), Vienna University of Technology, Austria, ISBN 3-9501554-0-6, July 7-12, 2002. Available from <http://wccm.tuwien.ac.at>.

[14] J.W. Hutchinson, Fundamentals of the phenomenological theory of nonlinear fracture mechanics, J. Appl. Mech. 50 (1983) 10421051.

[15] S. Li, W.K. Liu, Numerical simulation of strain localization in inelastic solids using mesh-free methods, Internat. J. Numer. Methods Engrg. 1 (48) (2000) 1285-1309.

[16] N. Moës, J. Dolbow, T. Belytschko, A finite element method for crack growth without remeshing, Internat. J. Numer. Methods Engrg. 46 (1999) 131-150.

[17] N. Moës, N. Sukumar, B. Moran, T. Belytschko, An extended finite element method (X-FEM) for two- and three-dimensional crack modelling, in: ECCOMAS 2000, Barcelona, Spain, September 11-14, 2000, Vienna University of Technology, Austria, ISBN 3-9501554-0-6.

[18] J. Mosler, G. Meshke, 3D FE analysis of cracks by means of the strong discontinuity approach, in: ECCOMAS 2000, Barcelona, Spain, September 11-14, 2000, Vienna University of Technology, Austria, ISBN 3-9501554-0-6. 
[19] J. Oliver, Modeling strong discontinuities in solid mechanics via strain softening constitutive equations. Part 1: Fundamentals, Internat. J. Numer. Methods Engrg. 39 (21) (1996) 3575-3600.

[20] J. Oliver, Modeling strong discontinuities in solid mechanics via strain softening constitutive equations. Part 2: Numerical simulation, Internat. J. Numer. Methods Engrg. 39 (21) (1996) 3601-3623.

[21] J. Oliver, On the discrete constitutive models induced by strong discontinuity kinematics and continuum constitutive equations, Internat. J. Solids and Structures 37 (2000) 7207-7229.

[22] J. Oliver, Topics on failure mechanics, Technical Report, International Center for Numerical Methods in Engineering (CIMNE), Monograph no. 68, December 2002.

[23] J. Oliver, M. Cervera, O. Manzoli, On the use of strain-softening models for the simulation of strong discontinuities in solids, in: R. de Borst, E. van der Giessen (Eds.), Material Instabilities in Solids, John Wiley, 1998, pp. 107-123 (Chapter 8).

[24] J. Oliver, M. Cervera, O. Manzoli, Strong discontinuities and continuum plasticity models: the strong discontinuity approach, Internat. J. Plasticity 15 (3) (1999) 319-351.

[25] J. Oliver, A. Huespe, E. Samaniego, A study on finite elements for capturing strong discontinuities, Internat. J. Numer. Methods Engrg. 56 (2003) 2135-2161.

[26] J. Oliver, A.E. Huespe, Continuum approach to material failure in strong discontinuity settings, Comput. Methods Appl. Mech. Engrg., submitted for publication.

[27] M. Ortiz, Y. Leroy, A. Needleman, A finite element method for localized failure analysis, Comput. Methods Appl. Mech. Engrg. 61 (1987) 189-214.

[28] N.S. Ottosen, K. Runesson, Properties of discontinuous bifurcation solutions in elasto-plasticity, Internat. J. Solids and Structures 27 (4) (1991) 401-421.

[29] R. Regueiro, R.I. Borja, A finite element model of localized deformation in frictional materials taking a strong discontinuity approach, Finite Elements Anal. Design 33 (1999) 283-315.

[30] J.R. Rice, The localization of plastic deformation, in: W.T. Koiter (Ed.), Theoretical and Applied Mechanics, North-Holland, 1976, pp. 207-220.

[31] J.G. Rots, Computational Modeling of Concrete Fractures, Thesis, Delft University of Technology, 1988.

[32] H.L. Schreyer, D.L. Sulsky, S.J. Zhou, Modeling delamination as a strong discontinuity with the material point method, Comput. Methods Appl. Mech. Engrg. 191 (2002) 2483-2507.

[33] J. Simo, J. Oliver, F. Armero, An analysis of strong discontinuities induced by strain-softening in rate-independent inelastic solids, Comput. Mech. 12 (1993) 277-296.

[34] G.N. Wells, L.J. Sluys, A new method, for modelling cohesive cracks finite elements, Internat. J. Numer. Methods Engrg. 50 (2001) 2667-2682.

[35] K. Willam, Constitutive models for engineering materials, in: Encyclopedia of Physical Science \& Technology, third ed., Academic Press, New York, 2000, pp. 603-633.

[36] O.C. Zienkiewicz, R.L. Taylor, The Finite Element Method, Butterworth-Heinemann, Oxford, UK, 2000. 